\title{
BULLETIN
}

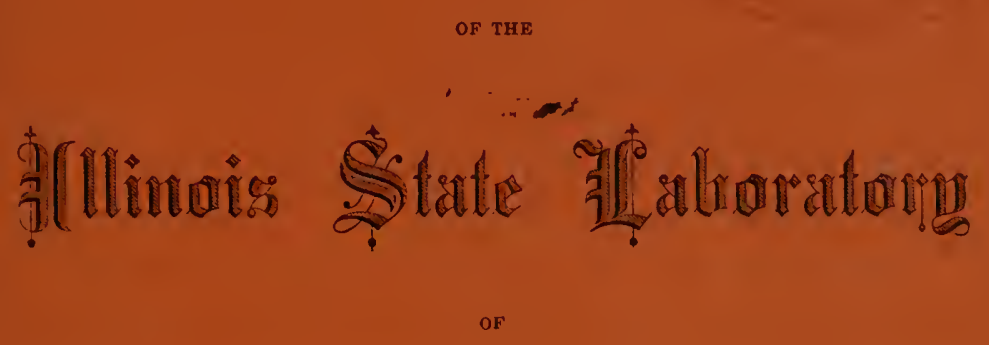

NATURAL HISTORY.

\section{CHAMPAign, ILlinois.}

\section{VOL UME II.}

ARTICLE Ir.-LIST of THE DESCRIBED SPEC'NES OF FRESH WATER CRUSTACEA From AMERICA, NoRTII OF MEXICO.

BY LUCIEN M. UNDERWOOD, PH. D.

1886.

J. W. FRANKS \& NONS, PRINTERS AND BINDERS.

PEORIA, ILLINOIS. 
Antrice V.- List of the Described Species of Fresh Wuter Crustace from America, North of Mexico. By Lueres II. Underwood, Рh.D.

\section{INTRODUCTORY.}

The economic relations of the fresh water Crustacea are rapidly calling them into general notice, since their importance as the natural food supply of many of. our most valuable fishes is established beyond question. It has seemed desirable that our scattered literature on this sulject should be indexed and made available to students. The following preliminary list is not intended as a complete arrangement of genera and species. A thorough revision of many of these groups must precede any permanent arrangement, and this is not possible at present. It is, therefore, merely an index to the described species, with such references to American and foreign literature as will place the student in a position to make use at once of all that pertains to each species. In case a full symonymy is given in standard works, like those of Leydig, Claus, P. E. Müller, Brady, etc., it has not been thought desirable to repeat this synonymy, but merely to refer to the anthor giving it in full. For this reason all the European papers are not repeated for all the species they describe. In the American literature, however, it has seemed desirable to give every reference to each species, even at the risk of too great repetition.

The study of our fresh water Crustacea, with that of their marine congeners, commenced with Thomas Say, and has since attracted the attention of over thirty different writers, nearly all of whom have added one or more new species to our lists. Certain groups, lilise the Phyllopode and Astacide, have been carefully monographed, and furnish a literature of great value; in others sufficient work has been accomplished to furnish a broad, if not a substantial, basis for further study; while in 
several others the ground has scarcely been broken, save by the brief and miscellaneous descriptions of new species. As in all such miscellaneous work there has been much looseness of definition and probably partial or complete re-description, many of the species as given in the following list will ultimately be reduced to synonyms or otherwise disappear from sight. While we deplore this looseness we cannot wholly ignore work because it has been poorly performed, as some of our recent carcinologists have been prone to do.

The species of fresh water Crustacea, as given herein, comprise 313 names, distributed in 88 genera and 29 families, as follows :

\begin{tabular}{|c|c|c|c|}
\hline & Families. & Genera. & Species. \\
\hline Coperoda ............................ & 8 & 14 & 66 \\
\hline 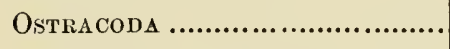 & 1 & 4 & 15 \\
\hline Cladocera $\ldots \ldots \ldots \ldots \ldots \ldots \ldots \ldots \ldots . . . . . . . . . .$. & 8 & 29 & 82 \\
\hline PIY LLOPODA............................ & 3 & 13 & 34 \\
\hline 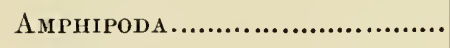 & 3 & 4 & 16 \\
\hline 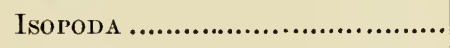 & 2 & 18 & 41 \\
\hline \multirow[t]{2}{*}{ 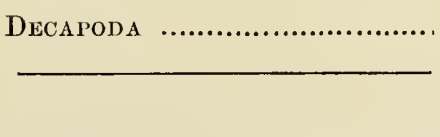 } & 4 & 6 & 59 \\
\hline & 29 & 88 & 313 \\
\hline
\end{tabular}

As to the relative abundance in the different sections of the country, too little is now known of the range of individual species to form any satisfactory conclusions. The following geographic table will, therefore, show the comparative amount of attention each group has received in different sectious rather than an exact representation of geographic distribution: 
'Table of Distribution of Species as knowy at tile piesent time.

\begin{tabular}{|c|c|c|c|c|c|c|c|c|}
\hline & 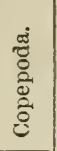 & 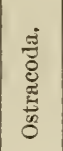 & 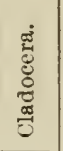 & 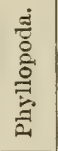 & 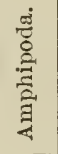 & 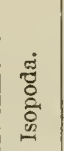 & 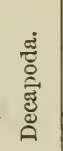 & 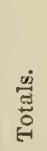 \\
\hline A labama .... & 7 & & 13 & & & & 12 & 32 \\
\hline California. & 1 & & & 1 & & 1 & 2 & $\begin{array}{r}1 \\
13\end{array}$ \\
\hline Colorado ............ & 1 & 3 & 2 & 4 & 3 & & & 13 \\
\hline ecticut ..... & 1 & & & 2 & 3 & 3 & & 9 \\
\hline Dakotal ........................... & & & & 1 & & & 2 & 3 \\
\hline District of Columbia.......... & & & & & & & 1 & 1 \\
\hline Florida............................... & ...... & 1 & ..... & 1 & 1 & & 5 & 8 \\
\hline ........................... & & 1 & ..... & $\cdots$ & 1 & 1 & 13 & 16 \\
\hline $\begin{array}{l}\text { Idali } \\
\text { Jlling }\end{array}$ & & & & & & & 1 & 1 \\
\hline Illinois $\ldots \ldots \ldots \ldots \ldots \ldots \ldots \ldots \ldots \ldots \ldots$ & 6 & .... & 2 & 2 & 5 & 6 & 9 & 30 \\
\hline 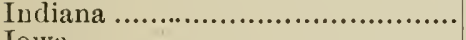 & 1 & ....... & $\cdots \cdots \cdot$ & 1 & 3 & 4 & 11 & 20 \\
\hline ..... & & ...... & ...... & 1 & $\cdots \cdot \cdots$ & ...... & & 7 \\
\hline ( & & & & 11 & & & 7 & 18 \\
\hline (n....................... & 4 & ....... & 3 & 1 & 1 & 1 & 7 & 17 \\
\hline Lou & 3 & ….. & …. & …... & & & & 8 \\
\hline (n.......... & 1 & ...... & ..... & ….. & 2 & ….. & & \\
\hline$\ldots \ldots \ldots \ldots, \ldots, \ldots, \ldots, \ldots, \ldots$ & & & & & & & 4 & 4 \\
\hline 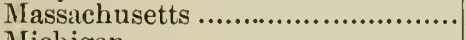 & 11 & 1 & 24 & 4 & 1 & 6 & 1 & 48 \\
\hline . . . & 4 & & & & 3 & 2 & 4 & 13 \\
\hline 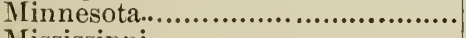 & 24 & 5 & 50 & 1 & & ...... & 2 & 82 \\
\hline n.................. & ... & ...... & 3 & ... & 1 & & & 13 \\
\hline Misso & & ...... & ..... & & .. & 1 & 7 & 8 \\
\hline (n)..................... & $\cdots \cdot$ & ..... & ….. & 2 & ...... & ...... & 1 & 3 \\
\hline ..... & & & & & & & 1 & 1 \\
\hline hire........ & & & & 1 & .... & & & 1 \\
\hline (2) & & 1 & ....... & 1 & .... & 2 & 4 & 8 \\
\hline ....... & & & & 1 & & & & 1 \\
\hline ( & 4 & 1 & 1 & 4 & 1 & 6 & 6 & $2: 3$ \\
\hline North Carolina & & & & & & & 4 & 4 \\
\hline Ohio ....... & 1 & ...... & & 3 & & & 4 & 8 \\
\hline ..... & & & & & & & 2 & 2 \\
\hline Pennsylval & & 4 & 2 & 3 & 1 & 4 & 2 & $16 j$ \\
\hline lihode Island & ・ & & & 2 & & & & 2 \\
\hline Carolina .................... & .... & …. & ..... & & & 1 & 10 & 11 \\
\hline Ten & & & & & 1 & 1 & 12 & 14 \\
\hline Texa & ... & & & 5 & & & 4 & 9 \\
\hline 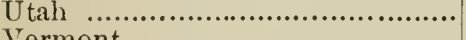 & & & & 3 & & & 1 & 4 \\
\hline nt ..... & & & & & $\cdots$ & & 1 & 1 \\
\hline & & & & & ...... & 1 & 4 & 5 \\
\hline Washington ................................. & 1 & & & & $\cdots \cdots$ & 2 & 4 & 7 \\
\hline West Virgir & & & & & & & 2 & 2 \\
\hline Wisconsin.. & 1 & ….. & 24 & 1 & 2 & & 6 & 34 \\
\hline , & ...... & ...... & ...... & 1 & ...... & ....... & 3 & 4 \\
\hline or $\ldots \ldots \ldots \ldots \ldots \ldots \ldots \ldots \ldots, \ldots$ & 2 & $\cdots \cdots \cdot$ & 5 & ...... & 4 & 1 & 5 & 17 \\
\hline an $\ldots \ldots \ldots \ldots \ldots \ldots \ldots \ldots$ & 4 & ….. & 8 & & 2 & & 1 & 15 \\
\hline & & & & & 1 & 1 & 1 & 2 \\
\hline 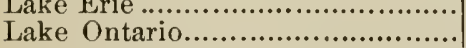 & 1 & ...... & ... & ..... $>>$ & . & ...... & & 1 \\
\hline British A & 3 & & & 1 & & 7 & 6 & 17 \\
\hline & & & & 3 & & 1 & & \\
\hline
\end{tabular}


In the above numbers Minnesota takes the lead with 82 species, owing to the extended study of the microscopic forms by Prof. C. I. Herrick. Massachusetts with 48 and Wisconsin with 34 owe much to the study of the Cladocera by Prof. E. A. Birge. Illinois with 30 , to which might properly be added most of the 15 of Lake Michigan, shows the careful research of Prof. S. A. Forbes. Other states owe their relatively high rank to the elaborate monograph of the Astacidæ, by Dr. Faxon, that has recently appeared. The contrast between the States above mentioned and others shows the nature of the work for future investigators. When we consider that twentyseven of the above States and territories do not exceed ten species (seventeen not exceeding five), and add Delaware, Nevada, Arizona, and Indian Territory, from which no species have been reported, we can begin to realize how meagrely the group has been studied. There ought to be a response by sonse one, or by many, from all these neglected regions.

Of the seven orders the Copepoda,* Ostracoda and Cladocera, including all the microscopic forms, have been studied only in the eastern half of the United States, if we except six species reported from Colorado by Chambers. In sharp contrast with this, 20 of the 34 Plyllopoda are known only west of the Mississippi River. Of the 17 Amphipoda four extend to Colorado, five to the Southern States, while the remainder seem confined to northern waters. The Isopoda and Decapoda are more uniformly distributed, and with the Phyllopoda extend to the Pacific coast.

The largest genera are Cambarus, with 50 species, Cyclops with 21 species, Daphnia with 15 species, Porcellio with 15 species, Cypris with 11 species, all the rest falling below 10 . Forty-one of the 88 genera are represented by a single species.

In the preparation of this list the compiler has been greatly aided by kind-hearted zoölogists who have furnished copies of their papers, cited references, corrected synomymies, and otherwise contributed to the completeness of the list. Their generosity entitles them to public thanks by the compiler and by all who may use this list when published. Their names

*This resnark does not properly apply to the parasitic forms, as some species have been reported from the Pacific coast. 
need not be mentioned here, for they comprise the entire number of living American writers, whose names appear in the following pages.

Syracuse University, April $1,1886$. 


\section{List OF THE SPECIES.}

\section{ORDER COPEPODA.}

\section{FAMILY CALANID无.}

I. Diaptomus Westw.

1. D. aRmatus Herrick. 10th Rep. Geol. Minn. 233 (1882); Final Report, 139 (1884).

Hab.-Minnesota (Herrick).

2. D. кentuckyensis Chambers. Jour. Cincinnati Soc. Nat. Hist. iv, 48 (1881).

Hab.-Kentucky (Chambers).

3. D. Leptopus Forbes. Amer. Nat. xvi., 646 (1882).

D. longicornis, var. leptopus Herrick. Final Report, 140, 186 (1884).

Hab.-Massachusetts and Illinois (Forbes).

4. D. Longrconnis Herrick. 7th Rep. Geol. Minn. 90 (1879); Final Report, 140, 141 (1884).

D. castor Herrick. 10th Rep. Geol. Minn. 221 (1882); Amer. Nat. xiii, 624 (1879).

Hab.-Minnesota (Herrick).

5. D. minnetonka Herrick. Final Report, 138 (1884). Hab.-Minnesota (Herrick).

6. D. Pallidus Herrick. 7th Rep. Geol. Minn. 91 (1879); Amer. Nat. xiii, 383 (1879); Final Report, 142 (1884).

Hab.-Minnesota (Herrick). 
7. D. SAxgurveus Forbes. Bull. Ill. Lab. Nat. Hist., No. 1. 15 (18\%6); Amer. Nat. xvi, 647 (1882).-Gissler. Amer. Nat. xv, 689 (1SS1).-Herick. Amer. Nat. xvii, 382 (1S83): Final Report, 138 (1S84).

Hab.-Illinois (Forbes), New York (Gissler), Alabama and Minnesota (Herrick).

8. D. sicilis Forbes. Amer. Nat. xvi, 645 (1882). D. pallidus, var. sicilis Herrick. Final Report, 142 (1884). Hab.-Lake Michigan (Forbes), Minnesota (Herrick).

9. D. stagralis Forbes. Amer. Nat. xvi, 645 (1882). -Herick. Final Report, 139 (1884).

I. giganteus Herrick. 10th Rep. Geol. Minm. 222 (1882). Irab.-Illinois (Forbes), Minnesota (Herrick).

\section{EPISCHURA Forbes.}

1. E. Fudriatilis Herrick. Amer. Nat. xvii, 384 (1883); Final Report, 133 (1884).

Hab.-Alabama (Herrick).

2. E. Lucustris Forbes. Amer. Nat. xvi, 648 (1982). -Herick. Final Report, 131 (1884).

?Scopiphora vagans Pickering MS. in DeKay. Nat. Hist. N. I. vi, 62 (1S44).

Hab.-Lake Michigan (Forbes), L. Ontario (Pickering).

\section{LIMNOCALANUS SAR8.}

1. L. Macrurus Sars. Oversigt af de indenlandske Ferskvands Copepoder (1862).-Forles. Amer. Nat. xvi, 648 (1882). -Heriek. Final Report, 134 (1884).

Hub.-Lake Michigan (Forbes).

\section{OSPHRANTICUM Forbes.}

1. O. Labronectum Forbes. Amer. Nat. xvi, 645 (1882). -Herick. Final Report, 134 (1884).

Potamoichetor fucosus Herrick. 10th Rep. Geol. Mim. 224 (1882).

Hab.-Lllinois (Forbes), Minnesota (Herrick). 


\section{FAMILY CYCLOPID正.}

\section{CyClops MÜlL.}

1. C. AqIuIs Koch. Deutschlands Crustaceen h. xxi, t., 11 (1838).-Forbes. Amer. Nat. xvi, 649 (1882).

Hab.-Illinois (Forbes).

2. C. ater Herrick. 10th Rep. Geol. Minn. 228 (1882); Final Report, 145 (1884).

Hab.-Minnesota (Herrick).

3. C. BRevispinosus Herrick. Final Report, 148 (1884). Hab.-Minnesota (Herrick).

4. C. diaphanus Fischer. Bulletin de la Soc. Imp. de Moscow, xxvi, 93 (1853).-Herrick. Final Report, 160 (1884). Hab.-Minnesota (Herrick).

5. C. elongatus Claus. Die freilebenden Copepoden, 97, t. xi, figs. 1, 2 (1863)._-Cragin. Trans. Kansas Acad. Science (1883).-Herrick. Final Report, 144 (1884).

Hab.-Massachusetts (Cragin).

6. C. fmimiatus Fischer. Bulletin de la Soc. Imp. de Moscow, xxvi, 94 (1853).-Herrick. Final Report, 162 (1884).

C. crassicornis Herrick. 10th Rep. Geol. Minn. 232 (1882). Hab.-Minnesota (Herrick).

7. C. fluviatiurs Herrick. 10th Rep. Geol. Minn. 231 (1882); Final Report, 159 (1884).

C. magnoctavus Cragin. Trans.Kansas Acad.Science (1883). Hab.-Massachusetts (Cragin), Minnesota (Herrick).

8. C. insectus Forbes. Amer. Nat. xvi, 649 (1882). -Herrick. Final Report, 152 (1884).

Hab.-Illinois (Forbes), Minnesota (Herrick).

9. C. Modestus Herrick. Amer. Nat. xvii, 500 (1883); Final Report, 154 (1884).

Hab.-A labama (Herrick). 
10. C. maficularis Say. Jour. Phila. Acad. i, 141 (1817).-DeKay. Nat. Hist. N. Y. vi, 62 (1844).--IIervich. Final Report, 163 (1884).

Hab.-Southern States (Say).

11. C. rarus Herrick. 10th Rep. Geol. Minn. 22!! (1852); Final Report, 152 (1884).

Itab.-Minnesota (Herrick).

12. C. orthoxomes Sars. Forhandlinger VidenskahsSilskabeh, 1862, 241.--Herick. Final Report, 150 (1854).

?C. temuissimus Herrick. Amer. Nat. xvii, 64 (1883).

Hul.-Kentucky, Alabama, Minnesota (Herrick).

13. C. Parcus Herrick. 10th Rep. Geol. Minn. 229 (1882); Final Report, 148 (1884).

Hab.-Minnesota (Herrick).

14. C. Pectinatus Herrick. Amer. Nat. xvii, 499 (1883). Hab.-Alabama (Herrick).

15. C. Phaleratus Koch. Dentschlands Crustaceen h. xxi. t. 9 (1838).-Herrick. Final Report, 161 (188t).

C. adolescens Herrick. 10th Rep. Geol. Minn. 231 (1882).

C. perarmatus Cragin. Trans. Kansas Acad.Science (1883). Hab.-Massachusetts (Cragin), Minnesota (Herrick).

16. C. PUlcheldus Koch. Deutschlands Crustaceen, h. xxi, t. 2 (1838). - Cragin. Trans. Kansas Acad. Science (1883).

Hab.-Massachusetts (Cragin).

17. C. serrulatus Fischer. Bulletin de la Soc. Inıp. des Nat. de Moscow, xxiv, 423 (18\$1).-Hervicl:. 10th Rep. Geol. Minn. 230 (1882); Final Report, 157, 163 (188t).

C. pectinifer Cragin. Trans. Kansas Acad. Science (1883). ?C. setosus Haldeman Jour. Phila. Acad. viii, 331.

Hab.-Massachusetts (Cragin), Minnesota (Herrick).

18. C. Texurconsis Claus. Archiv. für Naturgeschichte 1857, 31, Tab. iii, figs. 1-11.-Herick. 10th Rep. Geol. Minn. 227 (1882); Final Report, 153 (1884).

C. signatus, var. fasciacomis Cragin. Trans. Kansas Acad. Science (1883).

Hub-Wassachusetts (Cragin), Alabama and Minnesota (IIrrick). 
19. C. thomasi Forbes. Amer. Nat. xvi, 649 (1882). -Cragin. Trans. Kansas Acad. Science (1883).--Herrick. Final Report, 153 (1884).

Hab.-Massachusetts (Cragin), Lake Michigan (Forbes).

20. C. uniangulatus Cragin. Trans. Kansas Acad. Science (1883).-Herrick. Final Report, 149 (1884).

Hab.-Massachusetts (Cragin).

21. C. vinidis Fischer. Bull. de la Soc. Imp. des Nat. de Moscow, xxiv 412, t. ix, figs. 1-11 (1851).-Cragin. Trans. Kansas Acad. Science (1883).--Herrick. Final Peport, 145 (1884).

C. ingens Herrick. 10th Rep. Geol. Minn. 228 (1882); Amer. Nat. xvii, 499 (1883).

Hab.-Massachusetts (Cragin), Alabama and Minnesota (Herrick).

\section{FAMILY HARPACTID仺.}

\section{Canthocamptus Westw.}

1. C. cavernarum Packard. Zoölogy, 298 (figure only). Hab.-Kentucky (Paskard).

2. C. Illunoisensis Forbes. Bull. Ill. St. Lab. Nat. Hist., No. 1, 14 (1876).-Herrick. Final Report, 170 (1884).

Hab.-Illinois (Forbes).

3. C. мinutus Baird. British Entomostraca, 204, tab. xxv, figs. 4-8, xxx, fig. 3 (1850).-Herrick. Final Report, 170 (1884).

C. minutus, var. occidentalis Herrick. Tth Rep. Geol. Minn. 95 (1879).

Hab.-Minnesota (Herrick).

4. C. minnesotensis Herrick. Final Report, 173 (1884). Ilab.-Minnesota (Herrick).

5. C. morthumbricus Brady, var. americanus, Herrick. Fiual Report, 170 (1884).

Hab.-Minnesota (Herrick). 
Described Species of Fresh Wreter Crustacen.

\section{TACHIDIUS.}

1. T. fonticola Chambers. Jour. Cincinuati Soc. Nat. Hist. iv, 47 (1S81).

IIıb.-Kentucky (Chambers).

\section{FAMILYERGASILID丑.}

I. ERgasilus Nornm.

1. E. Cextrarchidarum Wright. Proc. ('amadian Inst. i, 243 (1S82).

Hab.-Canada; on various Centrarchille (Wright).

2. E. Funder Kroyer. Naturhistorisk Tidsskrift, ii, 228, 238, pl. 11, fig. 1 (1S63).

Hab.-Lonisiana; parasitic on Fundulus fimbratus: (Kroyer).

FAMILY CALIGID丑,

1. LEPEOPHTHEIRUS NoRDM.

1. L. SAlyonis Kroyer. Naturhistorisk Tidsskrift, ii. 137 , pl. 17, fig. 1 (1863).-W Smith. Rep. U.S. Fish Com. for 1SS2-S3. 662.

Ifab.-Eastern U.S.; parasitic on the salmon.

\section{FAMILY ARGULID帅.}

\section{Argulus MULl.}

1. A. ALos.æ Gould. Invert. Mass., 340 (1S41).

Hab.-Massachusetts; parasitic on alewife (Gould).

2. A. Catostom Dana and Herrick. Amer. Jour. Science, 1st series, xxx, $38 S$ (1S36).-Smith. Rep. U.S. Fish Com. for $1872-73,662$.

Hab.- Yonnecticut; parasitic on Catostomus sp. (Dana). 
3. A. Funduli Kroyer. Naturhistorisk Tidsskrift, ii, 20, pl. 2, fig. 1 (1863).-Smith. Rep. U. S. Fish Com. for 1872-73, 662.

Hab. -Louisiana; parasitic on Fundulus limbatus (Kroyer).

4. A. LePidostei Kellicott. Bull. Buff. Soc. Nat. Sciences iii, 214 (1877); Amer. Jour. Mieros. iii, 1 (1878); iv, 153, (1879).

Hab.-Niagara River (Kellicott).

5. A. pugetensis Dana. Crustacea, 1351 (1852).

Hab.-Washington Territory (Dana).

6. A. strzostethir Kellicott. Amer. Jour. Micros. v, 53 (1880); N. A. Entomologist, i, 57 (1880).

Itab.-Niagara River (Kellicott).

\section{FAMILY LERNÆOCERID无.}

I. LERN mOCERA Blainv.

1. L. cruciata Le Sueur. Jour. Phil. Acad. iii, 286 (1824). DeKay. Nat. Hist. N. Y. vi, 59 (1844).-Smith. Rep. U. S. Fish Com. for 1872-73, 665.-Kellicott. Proc. Amer. Soe. Mieros. i, 64 (1879).

Hab. - Lake Erie (Le Sueur), Michigan (Kellicott). On Centrarchus ieneus.

2. L. сатовтомг Kroyer. Naturhistorisk Tidsskrift, ii, 321, pl. 18, fig. 4 (1863).-Smith. Rep. U. S. Fish Com. for 1872-73, 665 .

Hab.-Mississippi River (Kroyer). On Catostomus macrolepidolus.

3. L. Pectoralis Kellicott. Proc. Amer. Soc. Micros. iv, 77 (1882).

Hab.-Michigan (Kellicott).

4. L. ромотіDis Kroyer. Naturhistorisk Tidsskrift, ii, 323, pl. xv, fig. 5 (1863).

Hab.-Louisiana (Kroyer). On Pomotis sp.

5. L. Tortua Kellicott. Proc. Amer. Soc. Micros. ii, 41 (1880).

Hub.-Tributaries of Lake Ontario, New York (Kellicott). 


\section{FAMILY LERNÆOPODIDÆ.}

\section{ACHTHERES NoRnM.}

1. A. ambyoplitis Kellientt. Proc. Amer. Soc. Micros. iv, $76(1882)$.

Hab.-Michigan (Krllicott).

2. A. Carpenteri Parkard. ith Rep. U. S. Geol. Survey (Hayclen), 611 (1874).

$H a b$.-Colorado (Packard).

3. A. conpulentus Kellicott, Proc. Amer. Soc. Micros. iv, 75 (18S2).

IIub.-Michigan (Kellicott).

4. A. LAC: Kroyer. Naturhistorisk Tiddskrift, ii. 274, pl. 17, fig. 6 (1863).--Smilh. Rep. U.S. Fish Com. for 1872-7\%, 603.

Hab.-North America (Kroyer).

5. A. micropten Wright. P'roc. Canadian Institute, i, $2+19(1883)$.

Hub.-Canada ( Wright).

6. A. Prmelon Kroyer. Naturhistorisk Tidsskrift, ii, 222, pl. 1\%, fig. 5 (1863). -Smith. Rep. U. S. Fish Com. for 1872-7\%, 6162.

Hab.-Ohio River (Kroyer). On Pimelodus maculatus.

\section{Cauloxenus Cope.}

1. C. straics Cope. Proc. Phili. Acad. 1871, 297; Amer. Nat. vi, 411 (1872); Third and Fonth liep. Geol. Indiana, 167 (1872).-Pucliud. Fifth Rep. Peabody Acakl. Sci. 9t (1873). Smith. Rep. U.S. Fish Cous, for 1872-73, 665.

Hetb.-Indiana (Cope).

\section{LERN ÆIOPODA ḰROYER.}

1. L. Californiensis Dana. Crustacea, 1379 (1552).

Iteb.-California (Dena). On salmon. 
2. I. Coregoni Smith. Rep. U.S. Fish Com. for 1872-73, 664,697 .

Hab.-Lake Superior (Smith). On Coregonus allus.

3. L. Euwardsi Olsson. Prodrom. fauna Copepodarum parasit. Scand., 36 (1868).-Wright. Proc. Canadian Inst. i, 246 (1883).

Hab.-Canada (Wright).

4. L. Fontinalis Smith. Rep. U. S. Fish Com. for 187273,663 .

Hab.-Maine (Smith). On Salmo fontinalis.

5. L. Siscowet Smith. Rep. U. S. Fish Com. for 1872-73, $664,69 \%$.

Hab.-Lake Superior (Smith). On Salmo siscowet.

\section{ORDER OSTRACODA.}

\section{FAMILY CYPRIDIDÆ.}

I. CANDONA BAIRD.

1. C. elongata Herrick. 7th Rep. Geol. Minn. 113, (1879).

Hab.-Minnesota (Herrick).

2, C. onnata Herrick. 7th Rep. Geol. Minn. 113 (1879). Hab.-Minnesota (Herrick).

\section{Cypris MÜlL.}

1. C. Agruis Haldeman. Proc. Phila. Acad. 1841, 53.DeKay. Nat. Hist. N.Y. vi, 65 (1844).

Hab.-Pennsylvania (Huldeman).

2. C. Altissimus Chambers. Bull. U.S. Geol. Survey (Hayden) iii, 152 (1877).

Hab.-Colorado (Chambers).

3. C. discolor Haldeman. Proc. Phila. Acad. 1842, 166. Hab.-Pennsylvania (Haldeman). 
Described Specios of Fresh Wuter Crustucen. 336

4. C. graxdis Chambers. Bull. U. S. Geol. Survey (Hayden ) iii, 151 (18\%).

Hab.-Colorado (Chambers).

j. C. hispid DeKay. Nat. Hist. N. Y. vi, 64 (1814).

Ifab.-New York and New Jersey (De K'(iy).

6. C. moxs Chambers. Bull. U. S. Geol. Survey (Hayden) iii, $15 \% 3(187 i)$.

If ab.--Colorado (Chambers).

7. C. negrecta Herrick. 7th Rep. Geol. Minn. 112 (1879).

Hab.-Minnesota (Herrick).

S. C. scabra Haldeman. Proc. Plilia. Acad. 184:, 184.

Hab.-Massachusetts (Huldeman).

9. C. smplex Haldeman. Proc. Phila. Acad. 1841, 53.Dekay. Nat. Hist. N. Y. vi, 6e (1844).

Hab.-Pennsylvania (Haldemen).

10. C. vidua Mïller. \%oül. Dan. Prod. No. 23St (17ria): Entomostraca 5j, t. 4, figs. 7-9 (1785).-Merick. Fth Rep. Geol. Mimm. 112 (18\%9).

ILub.-Minnesota (Herrich).

11. C. vitrea Haldeman. Proc. Phila. Acad. 1St:, 166. Hab.-Pennsylvania (Haldeman).

\section{Notadromus Lilijeb.}

1. N. sonacul's Lilljeborg. De Crustaceis ex.orl. tribus, (5), t. viii, figs. 1-15 (1853).-Lervicli. 10th Rep. Geol. Mim. $252(1882)$.

(Ygnis monuchus Müller. Entomostraca, 60, t. i, figs. (i-8, $(1785)$

Hab.-Minnesota (Ifrrick).

\section{Cytherina Là.}

1. C. bifaschita Say. Jour. Phila. Acad. i, 439) (1817). Dekuy. Nat. Hist. N. Y. vi, 6j (18t4).

Hab.-Georgia and Florida (Sry). 


\section{ORDER CLADOCERA.}

\section{FAMILY SIDID粃.}

\section{Sida Strauss.}

1. S. crystallina Strauss. Mem. du Museum, t. vi (1820). -Birge. Trans. Wis. Acad. iv, 78 (1877).-Herrick. 7th Rep. Geol. Minn. 99 (1879); 10th Rep. Geol. Minn. 235 (1882); Final Report, 20 (1884).

Daphnia crystallina Müller. Entomostraca, 96, t. 14, figs. $1-4(1785)$.

Hab.-Massachusetts, Lake Michigan, and Wisconsin (Birge); Minnesota (Herrick).

\section{Pseudo-Sida Herrick.}

1. P. iidentata Herriek. Final Report, 20 (1884). Hab.-Alabama (Herrick).

\section{DAPHNELla Baird.}

I. D. BRACHyLra Baird: British Entomostraca, 109(1850). -Herrick. 10th Rep. Geol. Minn. 236 (1850); Final Report, $21(1884)$.

D. winchelli Herrick. 7th Rep. Geol. Minn. 122 (1879).

? D. exspinosa Birge. Trans. Wis. Acad. iv, 79 (1877).

Sida brachyura Lievin. Branchicpoden der Danziger Umgegend, 20, t. iv, figs. 3-9 (1848).

\section{Latona Strauss.}

1. L. Setifera Strauss. Mem. de Museum, v, (1820).Birge. Chicago Med. Jour. and Ex. xvi, 585 (1881).-Herrick. Final Report, 22 (1884).

Daphnia setifera Mïller. Entomostraca, 98, t. 14, figs. 5-7 (1785).

Hab.-Massachusetts and Lake Michigan (Birge). 


\section{FAMILY HOLOPEDID丑.}

\section{HOLOPEDIUM $/ \mathrm{ADD}$.}

1. H. gibberum Zaddach. Archiv. für Naturgeschichte, 1855, 159, t. viii, ix.-Forbes. Amer. Nat. xvi, 641 (18S2).Herrick. Final Report, 22 (1884).

Hab.-Lake Michigan (Forbes).

\section{FAMILY DAPHNID 仺.}

I. MOINA BATRI.

1. M. Rectinostris Baird. British Entomostraca, 101, t. xi, figs. 1, 2 (1S50).-Birge. Trans. Wis. Acad. iv, 79 (1877). Herick. 10th Rep. Geol. Mimn. 23i (1882); Final Report, 34 (1S84).

M. Wrachiata Baird l.c. 102, t. ix, figs. 1, 2.

Duphnia rectirostris Müller. Entomostraca, 92, t. 12, figs. $1,2(1785)$.

Hab.-Alabama and Minnesota (Herrick); Wisconsin (Birge).

\section{Ceriodaphinia Dina.}

1. C. nlabamensis Herrick. Amer. Nat. xvii, j0:3 (1S83): Final Report, 38 (1884).

Hab.-Alabama (Herrick).

2. C. consons Birge. Trans. Wis. Acad. iv, 81 (1877). -Herrick. Final Report, 40 (1884).

$H(a b .-W$ isconsin (Birge).

3. C. cristata Birge. Trans. Wis. Acad. iv, 82 (1877). Herrick. Final Report, 38 (1884).

? Dap)lnia reticulate Herrick. 7th Rep. Geol. Minn. 104 $(1879)$.

Hab.-Massachusetts and Wisconsin (Birge); Minnesota (Herrick).

5. C. Laticalodita P. E. Müller. Dänmarks Cladocera, 130. t. i, fig. 19 (1868).-Herrick. Final Report, 39 (1884).

Hab.-Minnesota (IIerrick). 
6. C. PARva Herrick. Amer. Nat. xvii, 504 (1883).

Hab.-Minnesota (Herrick).

7. C. scitula Herrick. Final Report, 40 (1884).

Hab.-Minnesota (Herrick).

\section{SCAPAOLEBERIS SCHÖDL.}

1. S. angulata Herrick. Amer. Nat. xvii, 502 (1883); Final Report, 44 (1884).

Hab.-Mississippi and Alabama (Herrick).

2. S. armata Herrick. 10th Rep. Geol. Minn. 243 (1882); Final Report, 43 (1884).

Hab.-Alabama and Minnesota (Herrick).

3. S. Aurita Birge in Herrick's Final Report, 175 (1884).

S. nasuta Birge. Trans. Wis. Acad. iv, 85 (1877).-Herrick: Final Report, 43 (1884).

Daphnia aurita Fischer. Bull. Naturforsch. Gesellsch. in Moscau, xxii (1849).

Hab.-Wisconsin (Birge).

4. S. mucronata Schödler. Branchiopoden der Umgegend von Berlin, 23 (1858).-Birge. Trans. Wis. Acad. iv. 84 (1877).-Herrick. 10th Rep. Geol. Minn. 241 (1882); Final Preport, 42 (1884).

Daphnia mucronata Müller. Entomostraca, 94, t. xiii, figs. 6, 7 (1785).-Merrick. 7th Rep. Geol. Minn. 104 (1879). IIab.-Eastern United States.

\section{SIMOCEPHALUS Schöbl.}

1. S. americanus Birge. Trans. Wis. Acad. iv, 82 (1877). -Hericl. Final Report, 47, 49 (1884).

Hab.-Mississippi and Minnesota (Herrick); Wisconsin (Birge).

2. S. Daphnomes Herrick. Amer. Nat. xvii, 503 (1883); Final Report, 48 (1884).

Hab.-Alabama (Herrick).

3. S. nostratus Herrick. Final Report, 47 (1883).

Hal.-Mississippi (Herrick). 
4. S. vetux's Schödler. Bramchiopoden der Umgegend von Berlin, 18 (1858).-Birge. Trans. Wis. Acad. iv, 82 (1874). -Hervick. 7th Rep. Geol. Minn. 103 (1879); Final Report, 46 (1S8t).

Daphne vetulu, Miiller. Zö̈l. Dan. Prod. No. 2399 (17i6). $H a b$. -Wisconsin (Birge), Minnesota (Hcrrick).

\section{Daphnia MÜll.}

1. D. a brupta Haldeman. Proc. Phila. Acad. $1842,184$. Hab.-Pennsylvania (Haldeman).

2. D. angulata Say. Jour. Phila. Acad. i, 440 (1817). DeKay. - Nat. Hist. N.Y. vi, 6j (1S44).

$H a b .-$ Southern States $(S(y)$.

3. D. Bizevicauin Chambers. Bull. U. S. Geol. Survey (Hayden) iii, 154 (187\%).

Hab.-Kentucky and Colorado (Chambers).

4. D. nubi Herrick. Amer. Nat. xvii, 501 (1883); Final Report, 61 (188t).

Hab.-Minnesota (Herruck).

5. D. galeata Sars. Om en Sommeren 1862, foretagen zoologisk Reise i Christianias og Trondhjems Stiftor, 21 (1863). P. E. Mïller. Naturhistorisk Tidsskrift, v, 117, pl. i, fig. 6 (1868).-Smith. Rep. U.S. Fish Com. for 1872-73, 695.-Herrick. Final Report, 61 (1884).

Hab.-Minnesota (Herrick); Lake Superior (S'mith).

6. D. nyalina Leydig. Naturgeschichte der Daphniden, 151, t. i, figs. 8-10 (1860).-Forles. Amer. Nat. xvi, 642 (1882). -INerrick. Final Report, 60 (1884).

D. longispina Herrick. Amer. Nat. xvii, 501 (1883).

D. pellucida P. E. Mïller. Naturhistorisk Tidsskrift, v. 116, pl. 1, fig. õ (1868).-Smith. Rep. U.S. Fish Com. 1872-73, 696.

Hub.-Kentucky and Minnesota (Herrick), Illinois and Lake Michigan (Forbes), Lake Superior (Smith). 
7. D. Kalbergensis Schödler. Die Cladocera des frischen Huffs, 18 t. i, figs. 1-3 (1863).-Herrick. Final Report, 63 (1884).

Hab.-Minnesota (Herrick).

8. D. kerusses Cox. Amer. Monthly Micros. Jour. iv, 88 (1883).-Kéllicott. Proc. Amer. Soc. Micros. vi, 129 (1884).

Ifal, -Wisconsin (Cox), New York (Kellicott).

9. D. magniceps Herrick. Final Report, 64 (1884). Hab.-Minnesota (Herrick).

10. D. minnehaha Herrick. Final Report, 59 (1884). IIab.-Minnesota (IIerrick).

11. D. Pulex Claus. Mem. de Museum, vii, 158 (1820).Smith. Rep. U.S. Fish Com. for 1872-73, 696.-Birge. Trans. Wis. Acad. iv, 87 (1877).-Merrick. 7th Rep. Geol. Minn. 101 (1879); 10th Rep. Geol. Minn. 238 (1882); Anter. Nat. xvii, 501 (1883); Final Report, 56 (1884).

IHab.-Massachusetts and Wisconsin (Birge), Alabama and Minnesota (Herrick), Lake Superior (Smith). 196.

12. D. Reticulata Haldeman. Proc. Phila. Acad. 1843,

Hal.-Pennsylvania (Haldeman).

13. D. Retrocurva Forbes. Amer. Nat. xvi, 642 (1882). Hab.-Illinois (Forbes).

14. D. Rosea Sars. Om de i Omegn. af Christania forekom. Cladocera, 268 (1862).-Hewick. Final Report, 59 (1881). IIab.-Minnesota (IIerrick).

15. D. rotundata Say. Jour. Phila. Acad. i, 440 (181\%.) -DeKay. Nat. Hist. N. Y. vi, 65 (1844).

IIab.--Southern States (Say).

\section{FAMILY BOSMINID丑.}

I. Bosmina Batrit.

1. A. connuta Baird. Trans. Berwick Nat. Club, ii, 149 (-).-Birge. Trans. Wis. Acad. iv, 91 (1887).-Herrick. 10th Rep. Geol. Minn. 244 (1882). 
Monoculus cornutus Jurine. Hist. d. Monocles qui se trouvent anx environs de Geneve, 142, t. xiv, figs. 8-10 (1820).

IIah,-Massachusetts (Birge), Minnewota (IIerrick).

2. B. Loxfriostris Baird. Ann. and Mag. Nat. Hist. xvii. 112 (-): British Entomostraca. 10j, t. xr, fig. 3 (1550).Birge. Trans. Wis, Acad. iv, 91 (18;6).-Mevicl: 7th Rep. Grol. Minn. 109 (1SiS); 10th Rep. Geol. Minn. 24t (1SS2).

Lynnceus longirostris Miiller. Entomostraca, 76, t. x, figs. 7, $8(1785)$.

IIah.-Massachusetts and Wisconsin (Birge), Minnesota (Iferrick).

3. B. strutat Herrick. 10th Rep. Geol. Minn. 32 (1582); Final Report, 66 (1884).

IIal.-Minnesota (IIerrick).

\section{FAMILY LYNCODAPHNID王.}

\section{MACROTHRIX Batrn.}

1. M. pađpfr Herrick. 10th Rep. Geol. Minn. 245 (1S82); Final Report, 70 (1S54).

IIal._-Minnesota (Herrick).

2. MI. Rosed Baird. Trans. Berwick Nat. Club, ii, 149 (-); British Entomostraca, 104 (1850).-Birge. Trans. Wis. Acad. iv, 90 (157\%).-Herrick. Final Report, 69 (1S54). IIal., W Wisconsin (Birge.)

3. M. Thesuconsis Kurz. Dodekns nener Cladocera, 26, t. iii. fig. 1 (15it).-Hemicl. 10th Rep. Geol. Minn. 245 (18s2); Final Report, 70 (18S4).

M. argilis Herrick. 7th Rep. Geol. Minn. 106 (1S79). IIal,-Ninnesota (IIerick).

\section{LAthonURA LiLLueb.}

1. L. Rectrostris Lilljeborg. De Crustaceis ex ordinis tribus, 57, t. iv, figs. 8-11, t. v, fig. 2, t. xxiii, figs. 12-13 (1853). -Birge. Trans. Wis. Acad. iv, S! (18\%).-Hervicli. Final lieport, 71 (1584).

Drephnir rectirostris Müller. Entomostraca, 92, t. xii. figs. 1-3 (175i).

IIal._-Minnesota (Herrick). 


\section{LYNCODAPHNIA HERRICK.}

1. L. мacrothroides Herrick. Amer. Nat. xvi, 1007 (1882); 10th Rep. Geol. Minn. 247 (1882); Final Report, 75 (1884).

IIal.-Minnesota (IIerrick).

\section{ILIOCRYptUS SARS.}

1. I. Spinifer Herrick. 10th Rep. Geol. Minn, 246 (1882); Final Report, 77 (1884). Bull. Scientific Lab. Denison University, i, 39 (1885).

Hal,-Minnesota (IIerrick).

\section{FAMILY LYNCEID Æ.}

\section{EURYCERCUS BAIRD.}

1. E. Lamellatus Bairil. Ann. and Mag. Nat. Hist. ii, S8, t. ii, figs. 1-S (1S43); British Entomostraca, 124, t. xv, fig. 1 (1850).-Smith. Rep. U. S. Fish Com. for 1872-73, 696.Birge. Trans. Wis. Acad. iv, 92 (187\%).-Herricl. 7th Rep. Geol. Minn. 122 (1879); 10th Rep. Geol. Minn. 248 (18S2); Final Report, S0 (1884).

Lynceus lamellatus Müller. Entomostraca, 73, t.,ix, figs. 4-6 (1785).

Iral.-Minnesota (IIerrick), Lake Superior (Smith).

\section{ACROperus BaIRD.}

1. A. Leucocephalus Schödler. Nene Beiträge zur Naturgeschichte der Cladoceren, 30, t, i, figs. 11-16 (1863).-Birge. Trans. Wis. Acad. iv, 109 (1877).-Herrick. Final Report, 81 (1884).

Lynceus leucocephalus Koch. Deutschlands Crustaceen, h. 36, pl. x (1838).

Mub.-Massachusetts and Wisconsin (Birge). 


\section{CAMPTOCERCUS BairI.}

1. C. Macrures Baird. Ann. and Mag. Nat. Hist. ii, 91, t. 3, figs. J, 6 (1843); British Entomostraca, 12s, t. xvi, fig. @ (1450).-Birge. Trans. Wis. Acail. iv, 109 (18\% $)$ ) - Memirli. ith Rep. Geol. Minn. 10\% (1879); 10th Rep. Geol. Minn. 249 (1SS2); Final Report, 84 (1SS4).

Lymreus macrurus Müller. Entomostraca, 77, t. 10, figs. $1-3(1755)$.

IIal,-Massachusetts, Wisconsin, and Lake Michigan (Birge), Minnesota (Herrick).

2. (!. Rotuxnts Herrick. 10th Rep. Geol. Mimm. 249 (1SS2): Final Report, st (1Sst).

Hal,-Dinnesota (Herrick).

\section{ALONOPSIS SARS.}

1. A. catissima Kinr. Dodekas nener Cladoceren, 40, t. ii, figs. 13-15 (157t).-Ifrvich: Final Report, 86 (1584).

IIal,-DMinnesota (IIerrick).

2. A. Menca Birge. Trans. Wis. Acad. iv. 108 (187\%).IIrrirli. Final Report, 86 (1S5t).

Mal.-Minnesota (Herrick).

\section{LEydigia Kunz.}

1. L. AciNthocerromes Línr. Dodekas nener Cladoceren, 53 (1874).-Herich: Final Report, 89 (1884).

L. quadranguluris Herrick. 10th Rep. Geol. Minn. 248 (1882).

Lynceus acanthocercoirles Fischer. Bull. Soc. Nat. de Moscon, 1S5ั4. 431, t. iii, figs. 21-2う.

II (wh,-Minnesota (Herrick).

2. L. quanRaxgutaris Kinr. Dodekas nener Cladoceren, j2. t. ii, fig. 1 (1574).-Hemirli. Final Report, SS (1S84).

Hal,-Alabama (IIerrick). 


\section{Graptoleberis Sars.}

1. G. Testuninaria Kurz. Dodekas neuer Cladoceren, 54, t. ii, figs. 11, 12 (1874).-Hevicl. Final Report, 90 (1884).

G. inermis Birge. Trans. Wis. Acad. iv, 102 (1877).Herrick. 10th Rep. Geol. Minn. 250 (1882).

Lynceus testudinarius Fischer. Bull. Soc. Nat. de Moscon, 1854, t. ix, figs. $1,2$.

IIal.- Massachusetts and Wisconsin (Birge), Minnesota (IIerricli).

\section{CREPIDOCERCUS BiRge.}

1. C. setiger Birge. Trans. Wis. Acad. iv, 102 (187y). -Herricli. 10th Rep. Geol. Minn. 250 (1882); Final Report, 91 (1884).

Hab.-Wisconsin (Birge), Minnesota (Herrick).

\section{ALONA BAIRD.}

1. A. Affinis Schödler. Neue Beiträge zur Naturgeschichte der Cladoceren, 19 (1883).-Mevicl. Final Report, 98 (1884).

Lynceus affinis Leydig. Naturgeschichte der Daphniden, 223 , t. ix, figs. 68,69 (1860).

IIab.-Minnesota (Herrick).

2. A. angulata Birge. Trans. Wis. Acad. iv, 104 (1877). -Herrick. Final Report, 95 (1884).

IIab.-Massachusetts (Birge).

3. A. glactalis Birge. Trans. Wis. Acad. iv, 106 (1877). -Herrick. Final Report, 100 (1884).

IIal.-Massachusetts (Birge).

4. A. uINeAta Schödler. Neue Beiträge zur Naturgeschichte der Cladoceren, 20, t. i, fig. 23 (1863).-Herrich. Final Report, 96 (1884).

Lynceus linertus Fischer. Bull. Soc. Nat. de Moscou, 1854, 429, t. iii, figs. 15,16 .

Iab.-Minuesota (Herricl). 
5.1. oblonga P. E. Miiller. Naturhistorisk Tidsskrift, r, 175, pl. iii, figs. 22, 23 (1\$68).-Birge. Trans. IV is. Aran. iv, 107 (15\%).-Herick. 10th Rep. Feol. Minn. 250 (1582): Final Report, $9 \%$ (1SSt).

IIah,_Wisconsin (Birge), Minnesota (IIerrick).

6. A. partela, var. temercelata Herrick. Final lieport, $100(1.584)$.

A. tuberculuta Kur\%. Dodekas nener Cladoceren, j1, t. ii, fig. 3 (15it).

\section{IIal,-Massachusetts (IIerrick).}

7. A. porrecta Birge. Trans. Wis. Acad. iv, 105 (15i7). -IIericli. Final Report, 99 (1S84).

IIab.-Massachusetts and Wisconsin (Birge).

S. A. quanraxgularts Baird. Ann. and Mag. Nat. Mist. ii, 92, t. 3, figs. 9-11 (1843); British Entomostracn, 131, t. xvi, fig. 4 (1559).-Merrick. Final Report, 9r (1884).

Lynceus quadrangularis Mäller. Entomostraca, 72, t. ix, figs. 1-3.-Herick. 7th Rep. Geol. Minn. 107 (1S79).

ILah,-Minnesota (IIerrick).

9. A. sangurea P. E. Müller. Naturhistorisk Tidsskrift, v, 177 (1S68).-Hervick. Final lieport, 95 (1S84).

Hall,-Minnesota (Herrick).

10. A. spixifera Schödler. Nene Beiträge zur Naturgeschichte der Cladoceren, 18, pl. i, figs, 17-22 (1873).-Birgfe. Trans. Wis. Acad. iv, 107 (1577).-Herrick. Final lieport, 99 (1584).

IIab.-M Massachusetts and Wisconsin (Birge).

\section{ALONELLA SARS.}

1. A. Excist Kurz. Dodekas nener Cladocesen, ว9 (1874). - Herick. Final Report, 103 (1S54).

?Pleuroxus insculptus Birge. Trans. Wis. Acad. iv, 95 (18\%).

Lynceus excisus Fischer.

IIal,-Massachusett, and Wisconsin? (Birge), Minnesota (Herrick).

2. A. pelchelda Herrick. Final Report, 103 (1854). Ilah.-Minnesota (Herrick). 
3. A. " PүgмæA Kurz. Dodekas neuer Cladoceren, 61, t. iii, fig. 7 (1874).-Herrick. Final Report, 105 (1884). IIal.-Minnesota? (Herrick).

\section{Pleuroxus Baird.}

1. P. acutirostris Birge. Trans. Wis. Acad. iv, 99 (1877).-Herrick. Final Report, 109 (1884).

IIab.-Massachusetts (Birge).

2. P. affinis Herrick. Final Report, 111 (1884). IIab.-Alabama (Herrick).

3. P. Denticulatus Birge. Trans. Wis. Acad. iv, 98, (1877).-Herick. Final Report, 110 (1884).

IIal._-Massachusetts, Lake Michigan, and Wisconsin (Birge), Minnesota and Alabama (IIerrick).

4. P. hamatus Birge. Trans. Wis. Acad. jv, 98 (1877). -Hemicli. Final Report, 110 (1884).

IIah.-Massachusetts (Birge), Alabama (Iterrick).

5. P. procurvus Birge. Trans. Wis. Acad. iv, 92 (1877). -Herricli. 10th Rep. Geol. Minn. 250 (1882); Final Report, 113 (1884).

IIal,-Massachusetts and Wisconsin (Birge), Minnesota (Herrick).

6. P. stramineus Birge. Trans. Wis. Acad. iv, 94 (1877). -Herick. Final Report, 108 (1884).

Hab.-Massachusetts (Birge).

7. P. unidens Birge. Trans. Wis. Acad. iv, 97 (1877). -Herrick: 10th Rep. Geol. Minn. 250 (18s2); Final Report, 111 (1884).

IIal.-Wisconsin (Birge), Minnesota and Alabama (Herrick).

\section{Chydorus Leach.}

1. C. cælatus Schödler. Nene Beitrïge zur Naturgeschichte der Cladoceren, 15, t. ii, fig. 44 (1863).-IIervich. Final Report, 117 (1884).

IIal.--Minnesota (Ilerrich). 
2. C. Globosus Baird. Ann. and Mag. Nat. Hist. ii, 90, t. 3, figs. 1-t (1S43); British Entomostraca, 127, t. xvi, fig. \%, (1S50).-Birge. Trans. Wis. Acad. iv, 100 (1877).-Memirli: Final Report, 116 (1SSt).

IIab.-Wisconsin (Birge), Minnesota (Herrick).

3. C. sph.æricus Baird. Ann. and Mag. Nat. Hist. ii, 89, t. 2, figs. 11-13 (1S43); British Entomostraca, 126, t. xvi, fig. S (1850).-Birye. Trans. Wis. Acal. iv, 99 (1877).-Clumbers. Bull. U.S. Geol. Survey (Hayden), iii, 15 (1875).Herick. Fth Rep. Geol. Minn. 108 (1879)) Final Report, 116 (1854).

IIal,-Massachusetts, Lake Michigan, and Wisconsin ( Riryr), Minnesota (IIerrick), Kentucky and Colorado (Chemblers).

\section{Monospilus Sars.}

1. M. mispar Sars. Crust. Cladoc. i Omgne af Christiana, 165 (1862).-- Hewirli. Final Report, 119 (1854); Bull. Scientific Laab. Denison Univ. i, 38 (1885).

Hab.-Minnesota (Herrick).

\section{FAMILY POLYPHEMID王.}

\section{POLYPhemus MULl.}

1. P. Pentertus De Geer. Mem. pour serv. a l'histoire Ins. yii, 467, t. 2S, figs. 9-13 (17\% ).-Birge. Trans. Wis. Acarl. iv, 109 (1STr).-Merricli. 10th Rep. Geol. Minn. 251 (1882); Final Report, 121 (1884).

Monoculus pediculus Limnarus. Syst. Nat. (12th Ed.) i, $1058(170 \%)$.

Polyphemus orulus Müller. Entomostraca, 119, t, 20, figs. $1-5(1785)$.

P. occidentalis Herrick. 7th Rep. Geol. Minn. 122 (1879). IIab.-Massachusetts (Birge), Minnesota (Herrick).

2. P. stafixals Herick. Final Report, 122 (1SS4).

Hub.-Minnesota? (Herrick). 


\section{FAMILY LEPTODORID丑.}

\section{LEPTODORA LILLIEB.}

1. L. HYALns Lilljeborg. Ofversigt af Vetens.-Akad. Förhandl., 1860, 265, pl. vii, figs. 1-22.-Smith. Rep. U. S. Fish Com. for 1872-73, 696.-Forbes. Amer. Nat. xvi, 641 (1852).-Merrich. Final Report, 123 (1854).

Hab.-Take Michigan (Forbes), Lake Superior (Smith).

\section{ORDER PHYLLOPODA.}

\section{FAMILY LIMNADID王.}

I. LimNetis Loven.

1. L. Brevifrons Packard. Bull. U. S. Geol. Survey (Hayden), iii, 172 (187\%); 12th Rep. U. S. Geol. Survey (Hayden), ii, 301 (1883).

IIab.-Kansas.

2. L. Gouldir Baird. Ann. and Mag. Nat. Hist. $3 \mathrm{~d}$ ser., $\mathrm{x}$, 393 (1862).-Packard. Fth Rep. U. S. Geol. Survey (Hayden), 618 (1874); Bull. U. S. Geol. Survey (Hayden), iii, 173 (1877); 12th Rep. U. S. Geol. Survey (Hayden), ii, 299 (1883).-IIerrick.

IIab.-New Hampshire, Massachusetts, Rhode Island, New York, Illinois, Canada.

3. L. GRACILICORNis Packard. Amer. Jour. Science, 3d ser., ii, 113 (1871); 7th Rep. U. S. Geol. Survey (Hayden), 618 (1874); 12th Rep. U. S. Geol. Survey (Hayden), ii, 302 (1883).

II ab.-Texas.

4. L. mucronatus Packard. Amer. Nat. ix, 312 (1S75); Bull. U. S. Geol. Survey (Hayden), iii, 172 (187r); 12th Rep. U. S. Geol. Survey (Hayden), ii, 300 (1S83).

IIab.-Kansas, Montana. 


\section{EstherIA Ro'pr.}

1. E. Belfrutiei Packard. Amer. Jour. Science, 3d. ser., ii, 11: (18\%1); 7th Rep. U. S. Geol. Survey (Hayden), 619 (187t); 12th liep. T. S. Geol. Survey (Hayden), ii, 309 (1883). IIal.-Texas.

2. F. Califonxica Packard. 6th Rep. Peabody Acal. Science, 5 j (1874): 7th Rep. U. S. Geol. Survey (Hayden), 618 (1874); 12th Rep. U. S. Geol. Survey (Hayden), ii, 304 (1883).

II al.-California.

3. E. compleximanus Packard. 12th Rej. U. S. Geol. Survey (Hayden), ii, 305 (1883).

Eulmumdia compleximume Packard. Bull. U. S. Geol. Surver (Hayden), iii, 174 (1877).

IIab.-Kansas.

4. E. roxesir Baird. Proc. Zoöl. Soc. London, 147, pl. xv, figs. 1a, 1b, 1c, 1d (1862).-Pacliarl. 7th Rep. U. S. Geol. Survey (Hayden), 619 (187t); 12 th Rep. U. S. Geol. Survey ( Hayden), ii, 310 (1883).

II (al, --Southern States? Cuba.

5. E. mexicana Clans. Beitrüge zur Kemnt. d. Entomostraken, t. iii, iv, figs. 33-54 (1860).-P'uckud. 12th Rep. U. S. (ieol. Survey (Hayden), ii, 306 (1883).

L. clarlii Packard. 6th Rep. Peabody Acad. Seience, 5j, (1874); Tth Rep. U. S. Geol. Survey (Hayden), 619 (1S74). Mexico.

IIch,-Lake Winnepeg, Kentucky, Ohio, Kansas, New Mexico,

6. E. Monsei Packard. Amer. Jour. Science, 3d ser., ii, 113 (1871); (ith Rep. Peabody Acad. Science, 56 (1874); 7th Rep. U.S. Geol. Survey (Hayden), 619 (1874); 12th liep. U. S. Geol. Survey (Hayden), ii, 308 (1883).

IIab.-Iowa, Dakota.

\section{Eulimnadia l'ackard.}

1. E. aftassizi Packard. 6th Rep. Peabody Aead. Scienre, 5t (187t); 7th Rep. U. S. Geol. Survey (Haylen). ti18 (1574); 12th liep. U. S. Geol. Survey (Hayden), ii, 311 (188:).

I/ab.-Penikese Island (Massachusetts). 
2. E. TexanA Packard. 12th Rep. U. S. Geol. Survey (Haylen), ii, 312 (1883).

Limnadiu texanu Packard. Amer. Jour. Science, 3d ser., ii, 111 (1871); 7th Rep. U. S. Geol. Survey (Hayden), 618 (1874).

IIal.-Kansas, Texas.

\section{Limnadella Girard.}

1. L. Corincen Haldeman. Proc. Phila. Acad. 1842, 184; Ibid. 1854, 34.-Paclard. \%th Rep. U. S. Geol. Survey (Hayden ), 618 (1874); 12th Rep. U. S. Geol. Survey (Hayden), ii, 313 (1883).

Unrecognizable from description.

Hab.-Pennsylvania (Haldeman).

2. L. Kiter Girard. Proc. Phila. Acad. 1854, 3.-Puckard. 12th Rep. U. S. Geol. Survey, ii, 311 (1883).

Unrecognizable from description.

Hab.-Ohio (Girard).

\section{LIMNADIA BroNG.}

1. L. americana Morse. Proc. Boston Soc. N. H., xi, 404 (1868).-Purlurd. Tth Rep. U. S. Geol. Survey (Hayden), 618 (1874); 12th Rep. U. S. Geol. Survey (Hayden), ii, 311 (1883).

IIab.-Massachusetts.

\section{FAMILY APODID画.}

I. APUS SCḦ̈FF.

1. A. Aqualis Packard. Amer. Jour. Science, $3 d$ ser., ii, 110 (1871); 7th Rep. U. S. Geol. Survey (Hayden), 620 (1874); 12th Rep. U.S. Geol. Survey (Hayden), ii, 320 (1883). IIab.-Kansas, Texas, Colorado?

2. A. mongicaudatus Le Conte. Annals N. Y. Lyceum, iv, 155 (1845).-Pacliard. 12th Rep, U. S. Geol. Survey (Hayden), ii, 324 (1883).

A. obtusus James. Long's Expedition, ii, 336 (1823).Puckard. 7th Rep. U. S. Geol. Survey (Hayden), 620 (1874). IIab.- Colorado, Wyoming, Kansas. 
3. A. Lucasants Packard. Amer. Jour. Science, 3d ser., ii, 109 (1871): 7th Rep. U. S. Geol. Survey (Hayden), 620) (1874): Bull. U. S. Geol. Survey (Hayden), iii, 179 (1877); 12th Rep. I. S. Geol. Survey (Hayden), ii, 321 (1883).

Ilab.-Kansas, Lower California.

4. A. Newberizi Packard. Amer. Jour. Science, 3d ser., ii, 109 (18\%1); ith Rep. U. S. Geol. Survey (Iayden), 620 (1874); 12th Rep. U. S. Geol. Survey (Hayden), ii, 321 (1883). II $a$ b. - - Utah.

\section{LEPIDURUS LEACH.}

1. L. Bhlobatus Packard. Bull. U. S. Geol. Survey (Iay(len), iii. 178 (187\%); 12th liep. U. S. Geol. Survey (Hayden), ii, 318 (1853).

IIab.-Colorallo.

2. L. cocesin Packard. Amer. Nat. ix, 311 (1815); Bull. U. S. Geol. Survey (Hayden), iii, 177 (1877); 12th liep. U. S. Geol. Survey (Hayden), ii, 317 (1883).

II $a b$. - Utah, Montana.

3. I. GLardatis Kroyer. Natmrhistorisk Tidsskrift, ad ser., ii, 431 (1847).-Puclurel. 7th Rep. U. S. Geol. Survey (Hayden), 619 (1874); 12th liep. U.S. Geol. Survey (IIayden), ii, 316 (1883).

IIab.-Arctic America, Greenland.

\section{FAMILY BRANCHIPODID平.}

\section{ARTEMIA LEACH.}

1. A. aracilis Verrill. Amer. Junr. Seiente, $2 \mathrm{~d}$ ser.,

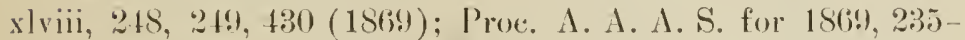
238.-Pucliurel. Tth liep. U. S. Geol. Survey (Hayden), (620), 621 (1874); 12th Rep. U. S Geol. Survey (Hayden), ii, 330 (1883).

A. monicu Verrill. Amer. Jour. Science, 2d ser., xlviii, $24 S(1869)$.

1. fertilis Verrill. Amer. Jour. Science, 2 d series., xlviii, $248(1869)$. 
A. utahensis Lockington. Monthly Micros. Jour. 1876 , 137, fide Packard.

II $a b .-$ Connecticut, Utah.

\section{BRANCHINECTA VERRILl.}

1. B. coloranensis Packard. 7th Rep. U. S. Geol. Survey (Hayden), 621 (1874); 12th Rep. U. S. Geol. Survey (Hayden ), ii, 338 (1883).

Hab.-Colorado.

2. B. Lindahli Packard. 12th Rep. U. S. Geol. Survey (Hayden ), ii, 339 (1883).

IIab.-Kansas.

3. B. Paludosa Packard. 12th Rep. U. S. Geol. Survey (Haydeu), ii, 336 (1883).

B. gronlandica Verrill. Amer. Jour. Science, 2d ser., xlviii, 253 (1869); Proc. A. A. A. S. for 1869, 244.-Packard. 7th Rep. Geol. Survey (Hayden), 621 (1874).

$B$. arctica Verrill. Amer. Jour. Science, $2 d$ ser., xlviii, 253 (1869); Proc. A. A. A. S. for 1869, 244.-Pacletel. 6th Rep. U. S. Geol. Survey (Hayden), 621 (1874).

Branchipus paludosus Mïller.

IIab.-Labridor, Arctic America, Greenland.

\section{BRalichipus Schüff.}

1. B. BUNing Forbes.

Éubranchipus bundyi Forbes. Bull. Ill. St. Lab. N. H., i, 25 (1876).

Hab.-Wisconsin (Forbes).

2. B. serratus Forbes.

Eubranchipus serratus Forbes. Bull. Ill. St. Lab. N. H., i, 13 (1876).--Packard. 12th Riep. U. S. Geol. Survey (Hayden ), ii, 343 (1883).

Hab.-Illinois (Forbes). 
3. B. vernalis Verrill. Amer. Jour. Seience, $2 d$ ser., xlviii. 25 (1S69): Proc. A. A. A. S. for 1869, 622.-Pachiorl. ith Rep. U. S. Geol. Survey (Hayden), 622 (15it): 12th liep. U. S. Geol. Survey (Hayden), ii, 342 (1583).-Gissler. Amer. Nat. xr, 136 (18s1).-Hay. Amer. Nat. xvi, 242 (1882).

Hab.-Massachusetts, Rhorle Island, Connecticut, New York, Pennsylvania, Ohio, Indiana.

\section{Chirocephalus Prevost.}

1. C. Holmaxi Ryder. Proc. Phila. Acad. 1879, 148.-Pacli"m. 12th Rep. U. S. Geol. Survey (Hayden), ii. 351 (188:3).Merrick: Bull. Scientific Lab. Denison Univ. i. 19 (1855).

Hal.-New York, Pennsylvania, Minnesota.

\section{Streptocephalus Baird.}

1. S. Floridaxts Packard. Amer. Nat. xiv, 53 (1850); 12th Rep. U. S. Geol. Survey (Hayden), ii, 350 (1883).

IIab.-Florida.

2. S. Sextil Ryder. Proc. Phila. Acad. 1879, 200.Parkard. 12th Rep. U. S. Geol. Survey (Hayden), ii, 348 (1S83).

Hab.-New York, New Jersey.

3. S. Texanus Packard. Amer. Jour. Science, 3rd ser., ii, 111 (1571); 7th Rep. U.S. Geol. Survey (Haylen), 622 (1574); 12th Rep. U.S. Geol. Survey (Hayden), ii, 345 (18s3).

S. Watsoni Packard. Bull. U. S. Geol. Survey (Hayden), iii, $176(187 \%)$.

Hub.-Kansas, Texas.

\section{Thamnocephalus Parkard.}

1. T. Platrunus Packard. Bull. U.S. Feol. Survey (Hayden), iii, 17 (15\% 15 ); 12th Rep. U. S. Grol. Survey (Hayden), ii, 353 (1553).

Ilab.-Kansas. 


\section{ORDER AMPHIPODA.}

\section{FAMILY GAMMARIDÆ.}

\section{Crangonyx Bate.}

1. C. antennatum Packard. Amer. Nat. xv, 880 (1881). IIab.-Tenuessee (Packard).

2. C. Bifurcus Hay. Amer. Nat. xvi, 144 (1882). IIab.-Mississippi (Hay).

3. C. gracilis Smith. Amer. Jour. Science, 3rd ser., ii, 453 (1871); Rep. U. S. Fish Com. for 1872-73, 654, 694.Forbes. Bull. Ill. State Lab. N. H. No. 1, 6 (1876).-Hay. Amer. Nat. xvi, 241 (1882).

IIab.-Indiana (Hay), Illinois (Forbes), Lake Superior ardd Lake Huron (Smith).

4. C. hucifugus Hay. Amer. Nat. xvi, 144 (1882). IIab.-Illinois (IIay).

5. C. mucronatus Forbes. Bull. Ill. State Lab. N. H., No. 1, 6 (1876).-Hay. Amer. Nat. xvi, 241 (1882).

IIab.-Indiana (IIay), Illinois (Forbes).

6. C. Packardir Smith. Amer. Jour. Science, 3rd ser., ix, 476-7 (1875).

C. vitreus Packard. 5th Rep. Peabody Acad. Science, 95 (1873).

Hab.-Indiana (Packard).

7. C. tenurs Smith. Rep. U. S. Fish Com. for 1872-73, 656.

Mab.-Connecticut (Smith).

8. C. vitreus Smith. Rep. U. S. Fish Com. for 1872-73, 656; Amer. Jour. Science, 3rd ser., ix, 476-7 (1875).

Stygobromus vitreus Cope. Amer. Nat. vi, 422 (1872); 3rd and 4th Rep. Geol. Indiana, 181 (1872).

IIab.-Mammoth Cave, Kentucky (Cope).

\section{Gammarus FabR.}

1. G. appendiculatus Say. Jour. Phila. Acad. i, 379 (1817).-DeKay. Nat. Hist. N. Y., vi, 37 (1844).

IIab.-Georgia (Say). 
2. G. Fasciatus Say. Jour. Phila. Acad. i, 379 (1817).DeKay. Nat. Hist. N. Y. vi, 37 (1844).-Smith. Rep. U. S. Fish Com. for 1572-73, 653.-Forbes. Bull. Ill. State Lab. N. H., No. 1, 6 (18;6).

Hab.--Maine (Verrill), Connecticut (Sinith), Penusylvania (Say), Illinois (Forbes), Michigan (Milner), Wisconsin (Verrill).

3. G. mmx.eus Smith. Rep. U.S. Fish Com. for 1872-73, 651, 694; 7th Rep. U. S. Geol. Survey (Hayden), 609 (1574).

G. lacustris Smith. Amer. Jour. Science, 3rd ser., ii, 453 (18\%1).

Hab.-Michigan, Lake Superior and Colorado (Smith).

4. G. mrus Say. Jour. Phila. Acad. i, 374 (181\%).-DeKay. Nat. Hist. N. I. vi, 37 (1844).—Gould. Invertebrata of Massachusetts, 334 (1841).-Smith. Rep. U.S. Fish Com. for $1872-73,654$.

Hab.-New York(?).

5. G. Robustus Smith. Fth Rep. U.S. Geol. Survey (Hayden ), $610(1874)$.

IIal.-Colorado (Smith).

\section{FAMILY LYSIANASSID尼.}

I. Pontoporeia Kröyer.

1. P. Filiconnis Smith. Rep. U. S. Fish Com. for 1872 -83, 649.-Forbes. Bull. Ill. State Lab. N. H., No. 1, 20 (1876).

Gammarus filicornis Stimpson MSS.

Hab.-Lake Michigan (Stimpson).

2. P. Hoy Smith. Rep. U. S. Fish Com. for 1872-73, 647, 694.-Forbes. Bull. Ill. State Lab. N. H., No. 1, 20 (1876).

P. affinis Smith. Amer. Jour. Science, 3rd ser., ii, 452 (1871).

Gammarus Hoyi Stimpson MSS.

Gammarns breristylus Stimpson MSS.

Hab.-Lake Michigan (Stimpson), Lake Suàerior (Smith). 


\section{FAMILY ORCHESTID丑.}

I. Allorchestes Heller.

1. A. dentatus Faxon. Bull. Mus. Comp. Zoöl., iii, 373, (1876).

Hyalella dentata Smith. Rep. U. S. Fish Com. for 187273, 645, 694; 7th Rep. U.S. Geol. Survey (Hayden), 611 (1874). -Forbes. Bull. Ill. State Lab. N. H.. No. 1, 5 (1876).

? Amphito Aztecus Saussure. Mem. sur divers Crust. nouv. du Mexique et des Antilles, 58 (1858).

?Allorchestes Knickerbockeri Bate. Cat. Amphip. Crust. Brit. Mus., 36 (1862).

IIab.-Maine, Massachusetts, Connecticut, Florida, Illinois, Wisconsin, Iowa, Michigan, Nebraska, Colorado, Oregon, Lake Superior.

Var. Inermis Faxon. Bull. Mus. Comp. Zoöl., iii, 373 (1876).

Hyalella inermis Smith. 7th Rep. U. S. Geol. Survey (Hayden ), 610 (1874).

Hab.-Colorado (Smith).

\section{ORDER ISOPODA.}

\section{FAMILY ASELLID止.}

\section{AseluUs Geoff.}

1. A. BRevicauda Forbes. Bull. Ill. State Lab. N. H., No. 1,8 (1876).

IIab.-Illinois (Forbes).

2. A. сомmunis Say. Jour. Phila. Acad., i, 428 (I817). -DeKay. Nat. Hist. N. Y., vi, 49 (1844).-Smith. Rep. U. S. Fish Com. for 1872-73, 65\%--Hay. Amer. Nat. xvi, 241 (1882).

?A. vulgaris Gould. Invertebrata of Massachusetts, 337 (1841).

A. militaris Hay. Bull. I1l. State Lab. N. H., No. 2, 90 (1878).

Hab.-Connecticut, Massachusetts, New York, Pennsylvania, Indiana, Illinois, Michigan, Mississippi. 
3. A. Ixtervedics Forbes. Bull. IIl. State Lab. N. H., No. $1,10(1876)$.

Hab.--Illinois (Forbes).

4. A. Lixeatus Say. Jour. Phila. Acad, i, 428 (1817).DeKoly. Nat. Hist. N. Y., vi, 50 (1844).

IIab.-South Carolina (Say).

5. A. straits Forbes. Bull. Ill. State Lab. N. H., No. 1, $11(18 ; 6)$.

Crecidotea stygia Packard. Amer. Nat., v, 751 (1871): 5th Rep. Peabody Acad. Science, 95 (1873).-Smith. Rep. U. S. Fish Com. for 18 22-73, 661.-Hubbard. Amer. Entomologist, n. ser., i, 36, 79 (1850).

C. microcephalu Cope. Amer. Nat., vi; 411 (1872); 3rd and 4th Rep. Geol. Indiana, 163 (1S72).-Smith. Amer. Nat., vii, $244(1873)$.

Hab.-Indiana (Cope), Illinois (Forbes), Kentucky (Packurd).

II. CACIDOTEA PACKARI.

1. C. Nickajackexsis Packard. Amer. Nat, xv, 879 (1S81).

IIab.-Tennessee (Packard).

\section{MANCASELLUS HaRger.}

1. M. Brachrunus Harger. Amer. Jour. Science, 3rd ser., xi, 304, $305(18 \div 6)$.

IIab.-Virginia (Harger).

2. MI. texax Harger. Amer. Jour. Science, 3rd ser., xi, 304 (1876).-Hay. Amer. Nat., xvi, 242 (1882).

Asellus tenax Smith. Amer. Jour. Science, 3rd ser., ii, 453 (1871).

Asellopsis tenax Harger. Amer. Jour. Science, 3rd ser., vii, 601 (18\%4).-Smith. Rep. U. S. Fish Com. for 1872-73, 6 อ๊ 9,695 .

Hab.-Indiana (Hay), Michigan and Lake Huron (Milner), Lake Superior (Smith). 


\section{FAMILY ONISCID仺.*}

\section{ACtoniscus Harger.}

1. A. eluipticus Harger. Amer. Jour. ร ;iea ce, 3rd ser., xv, 373 (1878); Proc. U. S. Nat. Museum, ii, 157 (1879); Rep. U. S. Fish Com. for 1878, 309.

Hab.-Connecticut (Harger).

\section{AlLoniscus Dana.}

1. A. Perconvexus Dana. Proc. Phila. Acad., vii, 176 (1854).

Hab.--California (Dana).

\section{ARMAdILlo Latr.}

1. A. speciosus Stuxberg. Öfversigt af Vetensk.-Akad. Förhandl., 1875, No. 2, 62.

Hab.-California (Stuxberg).

\section{ARMadillidium Brandt.}

1. A. PIlulare Stuxberg. Öfversigt af Vetensk.-Akad. Förhandl., 1875, No. 2, 63.

Armadillo pilularis Say. Jour. Phila. Acad., i, 432 (1817). -Gould. Invertebrata of Massachusetts, 336 (1841).-DeKay. Nat. Hist. N. Y., vi, 54 (1844). (Say).

Hal.-Massachusetts (Gould), New York (DeKay), Pennsylvania

\section{LigIA FABr.}

1. L. Dilatata Stimpson. Proc. Boston Soc. N. H., vi, 88 (1857); Boston Jour. Nat. Hist., vi, 507 (1857).

Hab.-Washington Territory (Stimpson).

2 L. occinentalis Dana. Crustacea, ii, 742 (1853).

Hab.-California (Dana).

* A few forms of Oniscidie are found only at or near the sea coast, and should properly take ramk among the marine species. The entire family, however, af known from America is indexed here. 
Described Species of Fresh Water Crustacen.

3. L. IPAldasm Brandt. Conspectus Oniscodorum, 172 (1833).

Hab.-Unalaska (Brandt).

\section{LIGIDIUM BRANIT.}

1. I. нурховим Budde-Iund. Naturhistorisk Tidsskrift, 3rd ser., vii, 225 (1870).-Stuxbery. Öfversigt af Vetensk.Akad. Förhandl., 1875, No. 2, 48.

Oniscus hypnorum Cuvier (1792).

Hab.-Niagara in Canada, and California (Eisen).

\section{ONISCUS L.}

1. O. Affinis Say. Jour. Phila. Acad., i, 430 (1817). Hab.-Pennsylvania (Say).

2. O. vicanius Stuxberg. Öfversigt af Vetensk.-Akad. Förhandl., 1872, No. 9, 3; Ibid. 18\%, No. 2. 50.

Hab.-Newfoundland (Lindahl), Canada near Niagara (Eisen).

\section{PhiLOscia Latr.}

1. P. spinosi Say. Jour. Phila. Acad., i, 429 (1817).DeKay. Nat. Hist. N. Y., vi, 50 (1884).

$H a b$.-Georgia (Say).

2. P. vitcata Say. Jour. Phila. Acad., i, 429 (1817).DeKay. Nat. Hist. N. Y., vi. 50 (1844).-Harger. Rep. U.S. Fish Com. for 1871, 569; Proc. U. S. Nat. Museum, ii, 157 (1S79): Rep. U. S. Fish Com. for 1878. 306.

Hab.-New Jersey, Connecticut and Massachusetts (Harger).

\section{ix. Euphiloscia Packard.}

1. E. Elronn Packard. 5th Rep. Peabody Acad. Science, 97 (1573).-Smith. Amer. Jour. Science, 3rd ser., ix, 477 (1875).

Hab.-Indiana (Packard). 


\section{Porcellio Latr.}

1. P. convexus Johnsson. Sveriges Oniscider, 32 (1868). - Stuxberg. Öfversigt af Vetensk.-Akad. Förhandl., 1875, No. 2,60 .

Oniscus convexus DeGeer (1778).

Hab.-Massachusetts, and Canada near Niagara (Eisen).

2. P. Dubius Brandt. Conspectus Oniscodorum, 178 (1833).

Hab.-? [This species is cited by Stuxberg loc. cit. 58.]

3. P. Fonmosus Stuxberg. Öfversigt af Vetensk.-Akad. Förhandl., 1875, No. 2, 57.

Hab.-California (Eisen).

4. P. gemmulatus Dana. Crustacea, 725, t. 47, fig. 7 (1853).

Hab.-Washington Territory (Dana).

5. P. glaber Fitch. Tran 3. N. Y. State Agric. Soc. 1854, 824; First and Second Rep. on Insects, 120 (1856).

Hab.-New York (Fitch).

6. P. immaculatus Fiteh. Trans. N. Y. State Agric. Soc. 1854, 824; First and Second Rep. on Insects, 120 (1856).

Hab.-Illinois and Arkansas (Fitch).

7. P. Limatus Fitch. Trans. N. Y. State Agric. Soc. 1854, 824; First and Second Rep. on Insects, 120 (1856).

Hab.-New York (Fitch).

8. P. Maculiconnis Koch. Deutschlands Crustaceen, 34, 16 (1849).-Stuxberg. Öfversigt af Vetensk.-Akad. Förhandl. 1875, No. 2, 55 .

Hab.-California (Eisen).

9. P. mixtus Fitch. 'Trans. N. Y. State Agric. Soc. 1854, 824; First and Second Report on Insects, 120 (1856).

Hab.-New York (Fitch).

10. P. Niger Say. Jour. Phila. Acad. i, 432 (1817).-DeKay. Nat. Hist. N. Y., vi, 52 (1844).

Hab.--Pennsylvania (Say). 
11. P. pictus Brandt. Conspectus Oniseolorum, 176 (1833).-Sturber\%. Öfversigt af Vetensk.-Akad. Förhandl., 1875. No. 2, 59 .

Hab.-Canada near Niagara (Eism).

12. P. scaber Latreille. Hist. Nat. Crust. et Insectes', vii, 45, in part (1804).-Fitch. Trans. N. Y. State Agrie. Soc. 1854, 825; First and Second Report on Insects, 121 (1856).Stuxberg. Öfversigt af Vetensk.-Akad. Förhandl. 1875, No. 2, 58 .

Oniscus asellus Limnens. Syst. Nat., Ed. 10, i, 637 (1758). -Gould. Invertebrata of Massachusetts, 326 (18t1)._De Kón. Nat. Hist. N. Y., vi, 51 (1844).

Hab.--Illinois (Fitch), Canada near Niagara, and California (Eisen), Newfoundland (Lindahl).

13. P. spinicornis Say. Jour. Phila. Acad., i, 431 (1817). Hab.--North America (Socy).

14. P. trilineatus Kioch. Dentschlands Crustaceen, 34, 9 (1840).-Stuxbery. Öfversigt af Vetensk.-Akad. Förhandl. 1875, No. 2, 59 .

Hub.--Mrassachusetts and Canada near Niagara (Eisen).

15. P. vitrafus Fitch. Trans. N. Y. State Agric. Soc. 1854, 814: First and Second Report on Insects, 120 (1856).

II $a b$. - New York (Fitch).

\section{Rhinoryctes Stuxberg.}

1. R. MirabiLis Stuxberg. Öfversigt, af Vetensk.-Akad. Förhandl. 1875. No. 2, 51.

Hab.-California (Eisen).

\section{SCYPHACELLA SiIth.}

1. S. arenicola Smith. Rep. U. S. Fish Com. for 1871, 568.-Harger. Proc. U. S. Nat. Museum, ii, 157 (187!)); Rep. U. S. Fish Cons. for 1S78, 307.

Hab.-Massachusetts and New Jersey (Harger). 


\section{SPH ÆrRILLO DANA.}

1. S. affinis Dana. Proc. Phila. Acad. vii, 176 (1854). Hab.-California (Dana).

\section{Styloniscus Dana.}

1. S. Gracilis Dana. Proc. Phila. Acad. vii, 176 (1854). Hab.-California (Dana).

\section{TRICHONISCUS BRANDT.}

1. T. Pusiluus Brandt. Conspectus Oniscodorum, 174 (1833)._Stuxbery. Öfversigt af Vetensk.-Akad. Förhandl. 1875, No. 2, 49.

$H a b .-C a n a d a$ near Niagara (Eisen).

\section{ORDER DECAPODA.}

\section{FAMILY MYSID丑.}

\section{MYSis Latr.}

1. M. ReLicta Loven. Öfversigt af Vetensk.-Akad. Förhandl. xviii, 285 (1861).-Smith. Amer. Jour. Science, 3rd ser., ii, 452 (1872); Rep. U. S. Fish Com. for 1872-73, 642, 694. -Forbes. Bull. Ill. State Lab. N. H., No. 1, 20 (1876).

M. diluvianus Stimpson MSS.

Hah.-Lake Michigan (Stimpson), Lake Superior (Smith).

\section{FAMILY ASTACIDÆ.}

\section{ASTACUS FABR.}

1. A. Gambellit Agassiz. Proc. Phila. Acad.vi, 375 (1853). -Stimpson. Jour. Boston Soc. N. H., vi, 492 (185\%); Proc. Boston Soc. N. H., vi, 87 (1857).-Hagen. Monog. Astacidæ, 90, pl. i, figs. 97, 98, pl. iii, fig. 170, pl. xi (1870).-Faxon. Proc. Amer. Acad. xx, 152 (1884): Revision of Astacidæ, 136 (1885).

Hab.-Utah, Idaho, Montana, Wyoming? California? 
2. A. KenMathexsis Stimpson. Proe. Boston Soc. N. H., vi, S7 (155i); Jour. Boston Soe. N. H., vi, 494 (185i).-Mayen. Monog. Astacidx. 93. pl. iii, 169 (18\%0).-Fuxon. Proc. Amer. Acad. xx, 1 jo (1SS4); Revision of Astacidae, 131, pl. vi, figs. 1, $\approx(1885)$.

Hab.-Oregon, Washington Territory, British Columbia.

3. A. Lexrescules Dana. Proc. Phila. Acad. vi, 20 (1852); Crustacea, i, 524, pl. xxxiii, fig. 1 (1852).-Stimpson. Jour. Boston Soc. N. H.. vi, 493 (1557): Proc. Boston Soc. N. H. 87 (15ã ).-Hagen.Monog. Astacide, 94 (15\%0).-Furon. Proc. Amer. Acad. xx, 151 (1884); Revision of Astacida, 132, pl. vi, fig. 4 (1885).

?A. Oreganus Randall. Jour. Phila. Acad. viii, 138, pl. vii (1839).-Stimpson. Proc. Boston Soc. N. H., vi, 87 (1852). -Hagen. Monog. Astacidie, 95 (1870).-Faxon. Revision of Astacidæ, 133 (18S5).

Hab.-Washington Territory

4. A. Nigrescexs Stimpson. Proc. Boston Soc. N. H., vi. 87 (185̃2); Jour. Boston Soc. N. H., vi, 492 (1952).-Hayen. Monog. Astacidæ, 92, pl. iii, fig. 168 (1870).-Furon. Proc. Amer. Acad. xx. 152 (1SSt); Rerision of Astacidæ, 135 (18S5).

Hab.-California, Washington Territory.

5. A. Trowbridgit Stimpson. Proc. Boston Soc. N. H., vi, 87 (1S5i); Jour. Boston Soc. N. H., vi, 492 (1S57).-Hagen. Monog. Astacidæ, 93, pl. iii, fig. 171, pl. x, (1870).-Faxon. Proc. Amer. Acad. xx, 152 (185t); Revision of Astacida, 134, (18S5̃).

Hab.-Oregon, Washington Territory.

\section{Cambarus Erich.}

1. C. Accminatus Faxon. Proc. Amer. Acad. xx, 113 (1S84): Revision of Astacida, 67. pl. iii, fig. 5. pl. viii, fig. ba, $6 \mathrm{a}^{\prime}(1855)$.

Hab.-North Carolina, South Carolina. 
2. C. abvena Hagen. Monog. Astacida, pl. iii, fig. 164, pl. viii (1870).-Faxon. Proc. Amer. Acad. xx, 140 (1884); Revision of Astacidæ, 54 (1885).

C. Carolinus Hagen. Monog. Astacidæ, 87, pl. i, figs. 5154, pl. iii, fig. 165 (1870).

Astacus advenc Le Conte. Proc. Phila. Acad. vii, 402 (1885).

IIab.-South Carolina, Georgia, Alabama.

3. C. affinis Girard. Proc. Phila. Acad. vi, 81 (1852). - Hagen. Monog. Astacidæ, 60, pl. i, figs. 19-22, 84, 85, pl. iii, fig. 152, pl. v (1870).—Faxon. Proc. Amer. Acad. xx, 146 (1884); Revision of Astacidæ, 86 (1885).

C. Pealei Girard, 1. c. 87 (1852).

Astacus affinis Say. Jour. Phila. Academy, i, 168 (1817). -Harlan. Med. and Phys. Res., 230, fig. 2 (1835).-DeKay. Nat. Hist. N. Y., vi, 22 (1844).

Hab.-New York, New Jersey, Pennsylvania, Maryland, District of Columbia, Virginia, Lake Erie, Lake Superior.

4. C. Alabamensis Faxon. Proc. Amer. Acad. xx, 125, 146 (1884); Revision of Astacidæ, 104, pl. iv, fig. 4, pl. x, figs. $3,3^{\prime}, 3 \mathrm{a}, 3 \mathrm{a}^{\prime}(1885)$.

Hab.-Alabama.

5. C. Aluent Faxon. Proc. Amer. Acad. xx, 110, 138 (1884); Revision of Astacidæ, 35, pl. i, fig. 1, pl. viii, figs. 2, $2^{\prime}$ (1885).

Hab.-Florida.

6. C. angustatus Hagen. Monog. Astacidæ, 50, pl, i, figs. 65-67, pl. iii, fig. 146 (1870).--Faxon. Proc. Amer. Acad. xx, 137 (1884); Revision of Astacidæ, 30 (1885).

Astacus angustatus Le Conte. Proc. Phila. Acad. vii, 401 (1885).

Hab.--Georgia.

7. C. argillicola Faxon. Proc. Amer. Acad. xx, 115, 144 (1884); Revision of Astacidæ, 76, pl. iv, fig. 2 (1885).

Hab.-Ontario, Michigan, Indiana, Louisiana? North Carolina? 
8. C. Bartonil Girard. Proc. Phila. Acad. vi, SS (1852). Hagen. Monog. Astacide, 75, pl, i, figs. 47-50, pl. ii, figs. 135139. pl. iii, fig. $166(1870)$.-Faxon. Proc. Amer. Acad. xx, 142 (1854); Revision of Astacidae, 59 (1855).

C. montanus Girard, l. c. 88 (1852).

?C. longulus Girard, l. c. 90 (1852).

?. pusillus Girard. 1. c. 90 (18522).

Astacus Bartonii Say. Jour. Phila. Acar. i, 16\% (1817).Harlan. Med, and Phys. Res. 230, fig. 3 (1535). - Gould. Invertebrata of Massachusetts, 330 (1841).—Thompson. History of Vermont. Part I, 170 (1842).-DeKay. Nat. Hist. N. Y., vi, 22, pl. viii, fig. 25 (1S44).

Hub.-New Brunswick, Province of Quebec? Irtine, Vermont, Massachusetts, New York, New Jersey, Maryland, Virginia, West Virginia, North Carolina, Ohio, Indiana, Kentucky, Tennessee, Lake Superior.

Var. Robusta Faxon. Revision of Astacidie, 61 (1855).

C. robustus Girard. Proc. Phila. Acad. vi, 90 (1852).Hagen. Monog. Astacidæ, 80, pl. iii, fig. 167 (1870).-Fiuxon. Proc. Amer. Acad. xx, 143 (1884).

Hab.-Province of Ontario, New York, Maryland, Virginia, Illinois.

9. C. Blandingir Hagen. Monog. Astacidae, 43, pl. i, figs. 63-64, pl. iii, fig. 140 (1870).-Faxon. Proc. Amer. Acad. xx, 136 (1S84); Revision of Astacidie, 19, pl. vii, fig. 2, 2', 2a, 2a', (1SS5).

C. acutus, var. B. Hagen, 1. c. pl. 36, iii, fig. 144 (1870.).

Astacus Blandingii Harlan. Trans. Amer. Philos. Soc. iii, 464 (1830); Med. and Phys. Res. 229, fig. 1 (1835)._? Le Conte. Proc. Phila. Acad. vii, 400 (185̃).

Hab. - New Jersey, Maryland, Virginia, North Carolina, South Carolina, Georgia.

Var. Acuta Faxon. Proc. Amer. Acad. xx, 136 (1884); Revision of Astacida, 20 (1885).

C. acutus Girard. Proc. Phila. Acad. vi, 91 (1852).Hagen. MLonog. Astacidæe, 35, pl. i, figs. 1-5, pl. ii, figs. 106, 108, 110-114, 116, 118, 120-124, 126, 127, pl. iii, fig. 143 (1870). -Forles. Bull. Ill. State Lab. N. H., No. 1, 3, 18 (1876).

C. acutus, var. A. Hagen, l. e. 36 , pl. ii, fig. 107, 109, 115, $117,119,125(1870)$. 
C. acutissimus Girard. Proc. Phila. Acad. vi, 91 (1852).

Hab.-Louisiana, Mississippi, Alabama, South Carolina, Tennessee, Missouri, Illinois, Indiana, Iowa, Wisconsin.

10. C. Carolinus Hagen (type).-Faxon. Proc. Amer. Acar. xx, 140 (1844); Revision of Astacidæ, 54 (1885). (1870).

C. adrena Hagen. Monog. Astacidæ, 86, pl. i, figs. 90-92

Hab.-Sonth Carolina.

11. C. Clankir Girard, Proc. Phila. Acad. vi, 91 (1852). -Hagen. Monog. Astacidæ, 39, pl. i, figs. 7-10, 99, 100, pl. ii, figs. 133, 134, pl. iii, fig. 142, pl. iv (1870).-Faxon. Proc. Amer. Acad. xx, 136 (1884); Revision of Astacidæ, 26 (1885).

Hab.-Texas, Louisiana, Mississippi, Alabama, Florida.

12. C. compressus Faxon. Proc. Amer. Acad. xx, 127, 146 (1884); Revision of Astacidæ. 105, pl. v, fig. 6, pl. x, figs. 2, 2', 2a, $2 \mathrm{a}^{\prime}(1885)$.

Hab.-Alabama.

13. C. connutus Faxon. Proc. Amer. Acad. xx, 120, 145 (1884); Revision of Astacidæ, 80, pl. v, figs. 1, 2, pl. ix, figs. $3,3^{\prime}(1885)$.

Hab.-Kentucky.

14. C. Diogenes Girard. Proc. Phila. Acad. vi, 88 (1852). -Faxon. Proc. Amer. Acad. xx, 144 (1884); Revision of Astacidæ, 71 (1885).

C. obesus Hagen. Monog. Astacidæ, 81, pl. i, figs. 39-42, pl. iii, fig. 163, pl. ix (1870).

Hab.-New Jersey, Maryland, District of Columbia, Virginia, North Carolina, Ohio, Indiana, Illinois, Michigan, Wisconsin, Iowa, Missouri, Kansas, Colorado, Wyoming, Arkansas, Kentucky? Mississippi, Louisiana.

15. C. pubius Faxon. Proc. Amer. Acad. xx, 114, 145 (1885); Revision of Astacidæ, 70, pl. iv, fig. 3, pl. viii, figs. 7, '7' (1885).

Hab.-Virginia, West Virginia, Tennessee.

16. C. extraneus Hagen. Monog. Astacidæ, 73, pl. i, figs. 88, 89, pl. iii, fig. 156 (1870).-Faxon. Proc. Amer. Acad. xx, 145 (1884); Revision of Astacidæ, 84 (1885).

Hab.-Georgia. 
17. C. Fallax Hagen. Monog. Astacidie, 45, pl. i, figs. 103-105 (1870).-Faxon. Proc. Amer. Acad. xx, 136 (1884); Revision of Astacida, 23, pl. ii, fig. 4 (1885).

Hub.-Florida.

18. C. Forceps Faxon. Proc. Amer. Acad. xx, 133, 148 (1884); Revision of Astacidie, 119, pl. v, fig. 4, pl. ix, figs. 5, 5', 5a, 5a' (1885).

Hab.-Alabama, Teunessee?

19. C. Girardianus Faxon. Proc. Amer. Acad. xx, 117, 145 (1884); Revision of Astacidie, 78, pl. iv, fig. 1, pl. ix, figs. 2a, $2 a^{\prime}(1885)$.

Hab.-Alabama.

20. C. gracrlis Bundy. Bull. Ill. State Lab. N. H., No. 1, 5 (1876); Trans. Wis. Acad. v, 182 (1882); Geology of Wisconsin, i, 403 (1883). - Faxon. Proc. Amer. Acad. xx, 141 (1884); Revision of Astacidre, 56, pl. viii, figs. 4, 4', 4" (1885); Bull. of Washburn Coll. Lab. N. H., i, 140 (1885).

Hab.-Wisconsin, Iowa, Illinois, Kansas.

21. C. Hamulatus Faxon. Proc. Amer. Acad. xx, 145 (1884); Revision of Astacidæ, 81, pl. iv, fig. 6, pl. ix, figs. 1, 1a' (1885).

Orconectes hamulutus Cope and Packard. Amer. Nat. xv, 881, pl. vii, figs. 1, 1a, 1b (1881).

Hab.-Nickajack Cave, Tennessee.

22. C. Harrisonit Faxoll. Proc. Amer. Acad. xx, 130, 147 (1854); Revision of Astacida, 94, pl. iii, fig. 1, pl. ix, figs. 9, 9' (1885).

Hab.--Missouri.

23. C. Hayr Faxon. Proc. Amer. Acad. xx, 108, 136 (1885); Revision of Astacide, 24, pl. i, fig. 4, pl. vii, figs. 3, 3', 3a, 3a' (1885).

Hab.-Mississippi.

24. C. Immunis Hagen. Monog. Astacidæe, 71, pl. i, figs. 101, 102, pl. iii, fig. 160, pl. viii, fig. b (1870).-Smith. Rep. U. S. Fish Com. for 1872-73, 639.-Forbes. Bull. Ill. State Lab. N. H., No. 1, 4, 19 (1876).-Bundy. Proc. Phila. Acad. 1877, 171.-F'axon. Proc. Amer. Acad. xx, 146 (1884); Revision of Astacida, 99, pl. $\mathrm{x}$, figs. 6a, 6a' (1885). 
C. signifer Herrick. 10th Rep. Geol. Minn. 253 (1882).

Hab.-New York, Indiana, Illinois, Michigan, Wisconsin, Minnesota, Iowa, Missouri, Kansas, Wyoming, Alabama, Mexico.

Var. Spinirostris Faxon. Proc. Amer. Acad. xx, 146 (1884); Revision of Astacidæ, 99, pl. 1, fig. 5 (1885); Bull. Washburn Coll. Lab. N. H., i, 140 (1885).

Hab.-Tenressee, Kansas.

25. C. Jordani Faxon. Proc. Amer. Acad. xx, 119, 145 (1884); Revision of Astacida, 83, pl. iii, fig. 3 (1885).

Hab.-Georgia.

26. C. LANCIFER ${ }^{-}$Hagen. Monog. Astacidæ, 59, pl. i, figs. 86, 87, pl. iii, fig. 159 (1870).-Fa.xon. Proc. Amer. Acad. xx, 146 (1884); Revision of Astacidæ, 86 (1885).

Hab.-Mississippi.

27. C. Latimanus Hagen. Monog. Astacidæ, 83, pl. i, figs. 43-46, pl. iii, fig. 162 (1870).-Faxon. Proc. Amer. Acad. xx, 144 (1884); Revision of Astacidæ, 69, pl. ii, fig. 3 (1885).

Astacus latimanus Le Conte. Proc. Phila. Acad. vii, 402 (1855).

Hab.-South Carolina, Georgia, Alabama, Mississippi, Tennessee.

28. C. Le Contei Hagen. Monog. Astacidæ, 47, pl. i, figs. 15-18, pl. iii, fig. 145 (1870).-Faxon. Proc. Amer. Acad. sx, 137 (1884); Revision of Astacidæ, 29, pl. ii, fig. 2 (1885).

Hab.-Georgia, Alabama.

29. C. maniculatus Hagen. Monog. Astacidæ, 52 (1870). -Faxon. Proc. Amer. Acad. xx, 137 (1884); Revision of Astacidæ, 29. (1885).

Astacus maniculatus Le Conte. Proc. Phila. Acad. vi, 91 (1855).

Hab.-Georgia.

30. C. medius Faxon. Proc. Amer. Acad. xx, 121, 145 (1884); Revision of Astacidæ, 107, pl. iii, fig. 4, pl. ix, fig. 4, 4' (1885).

Hab.-Missouri.

31. C. Mississippiensis Faxon. Proc. Amer. Acad. 123, 146 (1884); Revision of Astacidæ, 101, pl. iii, fig. 2, pl. x, figs. $4,4^{\prime}, 4 \mathrm{a}, 4 \mathrm{a}^{\prime}(1885)$.

Hab.-Mississippi. 
32. C. Nats Faxon. Bull. Washburn Coll. Lab. N. H., i, $140(1885)$.

$H a b$.-Kansas.

33. C. nebrascensis Girard. Proc. Phila. Acarl. vi, 91 (1852).-Hagen. Monog. Astacidx, 83 (1870).-Faxon. Proc. Amer. Acad. xx, 145 (1894); Revision of Astacida, 75 (1S85). Hab.-Dakota.

34. C. Neglectus Faxon. Bull. Washburn Coll. Lab. N. H., i, 142 (1S85).

\section{Hab.-Kansas.}

35. C. palmeri Faxon. Proc. Amer. Acad. 124, 146 (1S84); Revision of Astacidæ, 103, pl. iii, fig. 6, pl. x. figs. 5a, 5a' (18S5).

36. C. Pelludidus Girard. Proc. Phila. Acad. vi, 87 (1852).-Hagen. Monog. Astacidæ, 55, pl. i, figs. 68-71, pl. iii, fig. 148, pl. vi (1870).-Fuxon. Proc. Amer. Acad. xx, 139 (1S84); Revision of Astacidæ, 40 (1S85).

Astacus pellucidus Tellkampf. Arch. Anat. Physiol. 1. Wissensch. Med. 1844, 383.

Astucus (Cambailus) pellucidus Erichson. Archiv für Naturgesch., Jahrg. xii, Bd. i, 95 (1846).

Orconectes pellucidus Cope. Amer. Nat. vi, 410, 419 (1872); 31 and 4th Rep. Geol. Indiana, 162, 173 (1872).

Orconectes inermis Cope. Amer. Nat. vi, 410, 419 (1S72); $3 d$ and 4th Rep. Geol. Indiana, 162, 173 (1872).

Ilab.-Kentucky, Indiana.

37. C. Penicillatus Hagen. Monog. Astacidæ, 53, pl. i, figs. 93, 94 [95, 96?] pl. iii, fig. 149 (1870).-Faxon. Proc. Amer. Acar. xx, 138 (1884); Revision of Astacidæ, 36 (1885).

Astacus penicillatus Le Conte. Proc. Phila. Acad. vii, 401 (1855).

Hab.-Georgia, Mississippi? South Carolina?

38. C. Propinques Girard. Proc. Phila. Acad. vi, 88 (1852).--Magen. Monog. Astacidæ, 67, pl. i, figs. 34-38, pl. iii, fig. 153 (1870).-Faxon. Proc. Amer. Acad. xx, 147 (1884); Revision of Astacidie, 91 (1885).

Hab.-Provinces of Ontario and Quebec, New York, Indiana, Illinois, Michigan, Lake Superior, Wisconsin, Iowa. 
Var. Sanborni Faxon. Revision of Astacida, 91, pl. v, fig. 3, pl. ix, figs. 10, 10', 10a, 10a' (1885).

C. sanborni Faxon. Proc. Amer. Acad. xx, 128, 147 (1884). Hal.--Kentucky, Ohio.

Var. obscura Faxon. Revision of Astacidæ, 92 (1885).

C. obscurus Hagen. Monog. Astacidæ, 69, pl. i, figs. 72-75, pl. iii, fig. 154 (1870).-Faxon. Proc. Amer. Acad. xx, 148 (1884).

$H a b$.-New York.

39. C. pubescens Faxon. Proc. Amer. Acad. xx, 109, 137 (1884); Revision of Astacida, 31, pl. i, fig. 3, pl. viii, fig. 1a, $1 a^{\prime}(1885)$.

Hab.-Georgia.

40. C. putnami Faxon. Proc. Amer. Acad. xx, 131, 148 (1884); Revision of Astacidæ, 118, pl. v, fig. 5, pl. ix, figs. 6, $6^{\prime}, 6 a, 6 a^{\prime}(1885)$.

Hab.-Kentucky, 'Tennessee, Indiana?

41. C. rusticus Girard. Proc. Phila. Acad. vi, 88 (1852). -Hagen. Monog. Astacidæ, 71, pl. i, figs. 80-83, pl. iii, fig. 161 (18\%0).-Fuxon. Proc. Amer. Acad. xx, 148 (1884); Revision of Astacidæ, 108, pl. ix, figs. 8, 8', 8a, 8a' (1885). (1870).

C. placidus Hagen. 1. c. 65, pl. i, figs. 76-79, pl. iii, fig. 158

C. juvenilis Hagen. 1. c. 66, pl. i, figs. 29-33, pl. iii, fig. 157 (1870).

C. wisconsinensis Bundy. Bull. Ill. State Lab. N. H., No. 1, 4 (1876); Trans. Wisconsin Acad. v, 181 (1882); Geol. Wisconsin, i, 402 (1883).

Hab.-Pennsylvania, Ohio, Indiana, Illinois, Kentucky, Tennessee, Wisconsin, Iowa, Missouri, Texas, Lake Superior.

42. C. shufeldtil Faxon. Proc. Amer. Acad. xx, 134, 149 (1884); Revision of Astacidæ, 124, pl. vii, fig. 1, pl. x, figs. 8, 8', 8a, 8a' (1885).

IIab.-Louisiana.

43. C. simulans Faxon. Proc. Amer. Acad. xx, 112, 140 (1884); Revision of Astacida, 48, pl. i, fig. 12, pl. viii, fig. 3, $3^{\prime}, 3 \mathrm{a}, 3 \mathrm{a}^{\prime}$ (1885).

Hub.-Texas, Kansas. 
4. C. slodxi Bunly. Bull. I1l. State Lah. N. H., No. 1. 24 (1876); Proc. Phila. Acad. 1877, 172.-Faxon. Proc. Amer. Acad. xx, 147 (1854); Revision of Astacida, 86, pl. iv, fig. j, pl. x. figs. 1. 1', 1a, 1a' (1SSう).

\section{IIal.-Indiana, Kentucky.}

45. C. spiculffer Hagen. Monog. Astacidie, 48, pl. i, figs. 59-62, pl. iii, fig. 147 (1570).-Fuxon. Proc. Amer. Acad. xx, 138 (1884): Reviaion of Astacida, 33, pl. ii, tig. 5 (1885). Astrens spiculifu' Le Conte. Proe. Phila. Acad. vii, 401 (185j).

\section{Hub.--Georgia.}

46. C. sprnoses Bundy. Proc. Phila. Acad. 187\%, 173.Furon. Proc. Amer. Acad. xx, 14S (1884); Revision of Astacidax, 11j, pl. ix, figs. 7, 7', 7a, 7a' (1885).

IIub.-South Carolina, Georgia, Alabama.

* (. straius Bundy. Bull. Ill. State Lab. N. H., No. 1, 3 (1876); Trans. Wiscousin Acad. v, 180 (1882); Geology of Wisconsin, i, 402 (1883).-Frexon. Proc. Amer. Acad. xx, 140 (1874); Revision of Astacida, 56 (1885).

* C. турндobius Faxon. Revision of Astacida, 45 (1885).

C. stygius Joseph. Berliner Entom. Zeitschr. xxvi (1882).

47. ( troglonyes Hagen. Monng. Astacida, 41, pl. i, figs. 11-14, pl. ii, fig. 141 (1870).-Furm. Proc. Amer. Acad. xx, 136 (1884); Revision of Astacide $2 \tau$ (188j).

Astucus troglodytes Le Conte. Proc. Phila. Acad. vii, 400 (1855).

A. fossarm Le Conte, l. c. 401 (1855).

IIab.-Georgia, South Carolina.

48. C. Lhleri Faxon. Proc. Amer. Acad. xx, 116, 145 (1884); Revision of Astacidie, 7\%, pl. viii, figs. 8, 8', 8a, 8a (1885).

Hab.-Maryland.

49. C. versutus Hagen. Monog. Astacidæe, 51, pl. i, figs. 55-58, pl. iii, fig. 150 (1870).-Furon. Proc. Aner. Acad. xx, 138 (1884); Revision of Astacide, 34 (1855).

Hab.-Florida, Alabama.

* These species are doubtful, and hence excluded from the numbered list, in accordance with Faxon's revision. 
50. C. viritis Hagen. Monog. Astacidæ. 63, pl. i, figs. 23-28, pl. ii, figs. 128-132, pl. iii, fig. 155, pl. viii (1870).-Herrick. 10th Rep. Geol. Minn. 253 (1882). - Faxon. Proc. Amer. Acad. 147 (1884); Revision of Astacidæ, 96 (1885).

C. debilis Bundy. Bull. Ill. State Lab. N. H., No. 1, 24 (1876); Trans. Wisconsin Acad. v, 181 (1882); Geology of Wisconsin, i, 403 (1883).

C. conesi Streets. Bull. U. S. Geol. Survey (Hayden), iii, $803(1877)$.

Hab.-Canada, Dakota, Minnesuta, Wisconsin, Iowa, Nebraska, Wyoming, Kansas, Missouri, Illinois, Indiana, Tennessee, Texas, New York?

\section{FAMILY PAL必MONID丑.}

I. PAL Æmon Fabr., Stimpson.

1. P. onionis Smith. Rep. U.S. Fish Com. for 1872-73, 640.-Forbes. Bull. Ill. State Lab. N. H., No. 1, 5 (1876).Hay. Amer. Nat. xvi, 143 (1882).

Hab.-Indiana, Illinois, Mississippi, Mississippi River.

\section{PaL æamonetes Heller.}

1. P. Paludosa Kingsley. Proc. Phila. Acad. 1878, 97; Bull. Essex Inst. x, 65 .

P. exilipes Stimpson. Ann. N. Y. Lyceum N. H., x, 130 (1871).-Smith. Rep. U. S. Fish Com. for 1872-73, 641.Forbes. Bull. Ill. State Lab. N. H., No. 1, 5 (1876).-Hay. Amer. Nat. xvi, 144 (1882).

Hippolyte paludosa Gibbes. Proc. A. A. A. S., iii, 197 (1850).

Hab.-Ohio, Illinois, Michigan, Tennessee, South Carolina, Florida, Mississippi.

\section{FAMILY PEN丑ID丑.}

I. Pen æaUs Latr.

1. P. Braziliensis Latr. Nouv. Dict. d' Hist. Nat., 154, t. xxv.-Milne-Edwards. Hist. Nat. des Crust. ii, 414 (1837). -Stimpson. Arm. N. Y. Lyceum N. H., x, 132 (1871).Smith. Rep. U. S. Fish Com. for 1872-73, 642.

Hab.-New York, New Jersey. 


\section{BIBLIOGRAPHY.}

The following papers, which include the principal works relating to the Fresh Water Crustacea in general, contain references to species known to inhabit North America. It will be seen to include numerous paper's by Americans from Say's time to the present.

BAIRD (William). Natural History of the British Entomostraca. With 36 plates. London (1850).

Monograph of the Family Limniadx, a Faunily of Eutomostracous Crustacea. Proceedings of the Zoölogical Society of London, xvii, 84-90, (1849).

Monograph of the Family Branchiopodida, with a Description of a New Genus, ete. Proceedings of the Zoölogical Society of London, xx, 18-31 (1852).

Monograph of the Family Apodidie, a Family of Crustaceans belonging to the Futomostraca. Proceedings of the Zoölogical Society of London, xx, 1-8 (1852).

Birge (Edward A.) Notes on Cladocera. Trans. Wisconsin Acad. Science, iv, 77-109. 2 plates (1877).

Notes on Crustacea in Chicago Water Supply, with Remarks on the Formation of the Carapace. Chicago Med. Jour. and Examiner, xvi, 584-590, double plate (Dec. 1S81).

BRADY (George S.) Monograph of the Recent British Ostracoda. With 19 plates. Trans. Linnaan Society, xxvi (1868).

Monograph of the Free and Semi-parasitic Copepoda of the British Islands. 3 vols., with 96 plates. London (1878-1880).

BRANDT (J. F.) Conspectus Oniscodorum (1833).

BUDDE-LUND (G.) Danmarks Isopoda. In Naturhistorisk Tidsskrift, 3d series, vii (1860).

BUNDY (William F.) On the Cambari of Northern Indiana. Proc. Phila. Acad., 1877, 171-17\%. 
List of the Crustacea of Wisconsin, with Notes on Some New or Little-known Species. Trans. Wisconsin Acad. Science, v, 177-184 (1882).

The Crustacean Fauna of Wisconsin. Geology of Wisconsin, i, 402-405 (1883).

Chambers (Vactor T.) New Entomostraca from Colorado. Bull. U. S. Geol. Survey (Hayden), iii, 151-155, figs. 1-4 (1877).

Two new species of Entomostraca. Jour. Cincinnati Soc. Nat. Hist., iv, 47, 48, two plates (1881).

Claus (C.) Das Genus Cyclops, und seiner einheimischen Arten. Archiv für Naturgeschichte (1857).

Beiträge zm Kenntniss der Entomostraken, 1860.

-Die frei-lebenden Copepoden Deutschlands. With 37 plates. Leipzig (1863).

Cope (Edward D.) On the Fanna of the Wyandotte Cave. Proc. Phila. Acad. of Science (1871).

On the Wyandotte Cave and its Fauna. Amer. Naturalist, vi, 406-422 (1872).

-Report on the Wyandotte Cave and its Fauna. Third and Fourth Report of the Geol. Survey of Indiana, 152$182(1872)$.

COPE (E. D.) and PACKard (A. S., Jr.) The Fanna of the Nickajack Cave. Amer. Naturalist, xv, 872-882 (1881).

CRaGin (Francis W.) A Contribution to the History of the Fresh-Water Copepoda. Trans. Kansas Academy of Science, viii, plates i-iv (1882). Also separate, pp. 1-15.

Dana (James D.) Conspectus Crustaceorum, etc. Proc. Phila. Acad. Science, vi, 6-28 (1852).

-United States Exploring Expedition, under the command of Charles Wilkes, U. S. N., Vol. xiii, Crustacea. Parts $\mathrm{i}$ and ii, and folio Atlas (1852).

Catalogue and Descriptions of Crustacea Collected in California by Dr. John L. Le Conte. Proc. Phila. Acad. Science, vii (1854).

DANA (James D.) and HerRICK (E. C.) A New Species of Argulus. Notice in Amer. Jour. of Science and Arts, 1st series, $\mathrm{xxx}, 388-389$ (1836). 
DEGEER (Ch.) Memoires pour servir a l'Histoire des Insects. 8 vols. $(1752-17 \% 6)$.

DEKAY (James E.) \%oülogy of New York. Part vi, Crustacea (1844).

ERICHSON (IV. F.) Uebersicht iler Arten der Gattung Astacus. In Archir für Naturgeschichte (1846).

FAXON (Walter). Descriptions of New Species of Cambarus, etc. Proe. Amer. Acull of Arts and Sciences, xx, 107158 (1884).

A Revision of the Astacida. Memoirs of Museum of Comparative \%oülogy. pp. 186. With 10 plates (1885). -Preliminary Catalogne of the Crayfishes of Kansals. Bulletin of the Washburn College Laboratory of Natural History, i, No. 4, 14()-142 (1885).

A List of the Astacidat in the United States National Musemm. Proc. U.S. Nat. Mus,, 356-361 (1885).

FIscher (S.) Crustaceen in del Ung. von St. Petershurg ans den Ordungen der Branchiopoden und Entomostratceen (1S+S-1850).

Neue oder wenig gekamnte Daphnien Arten (1819-1S5̃1). -Ueber das genus Cypris (1851).

Beiträge zur Kenntniss der (yclopiden der Umg. von St. Petersburg (1851-1853).

Fiтch (Asa). First Report on Noxious Insects of New York. Trans. N. Y. State Agric. Society, 1854, 705-880. [Description of species of Porcellio, pp. 823-825.]

First and Second Reports on the Noxions [nsects of New York, 1856. [Description of species of Porcellio. pl. 116-121.]

FORBas (Stephen A.) List of Illinois Crustacea, with Descriptions of New Species. Bulletin of the State Laboratory of Natural History, i, No. 1, 3-25 (1876).

On some Entomostraca of Lake Michigan and Adjacent Waters. American Naturalist, xvi, 53\%-542, 640-65̃0 (1882).

GibBes (Lewis R.) ()n the Carcinological Collections of the United States. Proc. Amer. Association for the Advancenent of Science, iii, 16\%-201 (1850). 
GiRard (Charles). Revision of the North American Astaci, with Observations on their Habits and Geographical Distribution. Proc. Phila. Acad. of Science, vi, 87-91 (1852).

On a new Entomostracan of the Family Limnadidæ inhabiting the Western Waters. Proc. Phila. Acad. of Science, iv, 3 (1854).

GissLeR (Carl F.) Contributions to the Fauna of the New York Croton Water (1872).

Description of a Hermaphrodite Phyllopod Crustacean. American Naturalist, xv, 136-139 (1881).

Evidences of the Effect of Chemico-physical Influences in the Evolution of Branchiopod Crustaceans. Amer. Naturalist, xv, 280-282 (1881).

Variations in a Copepod Crustacean. Amer. Naturalist, xч, 689-698 (1881).

Gould (Augustus A.) Report on the Invertebrata of Massachusetts. Crustacea, pp. 11-23; 321-341 (1841).

HAGEN (Hermann A.) Monograph of the North American Astacidæ. Plates i-xi. Illus. Cat. Museum of Comparative Zoölogy, No. 3 (1870).

The Blind Crayfish. Amer. Naturalist, vi, 494 (1872).

Haldeman (Samuel S.) Two Species of Cypris. Proc. Phila. Acad. of Science, i, 53 (1841).

Description of Two New Species of Cypris. Proc. Phila. Acad. of Science, i, 166 (1842).

- Description of Two Species of Entomostraca. Proc. Phila. Acad. of Science, i, 184 (1842).

-Description of a New Species of Cypris. Proc. Phila. Acad. of Science, i, 184 (1842).

Description of Two New Species of Hydrachna, and One of Daphnia. Proc. Phila. Acad. of Science, i, 196 (1842).

[Note on Limnadella.] Proc. Phila. Acad. of Science, vii, 34 (1854).

Harger (Oscar). On a New Genus of Asellidæ. Amer. Journal of Science and Arts, 3d series, vii, 601, 602 (1874). 
Description of Mancasellus brachyurus, a New Freshwater Isopod. Amer. Jomrnal of Science and Arts, 3d series, xi, 304, 305 (1876).

Description of New (renera and Species of Isopoda from New England and Adjacent Waters. Amer. Journal of Science and Arts, 3d serles, xv, 373-379 (1878).

Notes on New England Isopoda. Proc. U. S. National Museum, ii, 15\%-165 (1879).

Report on the Marine Isopoda of New England and Adjacent Waters. Report of U.S. Commission on Fish and Fisheries for 1878, 297-462, Plates i-xiii.

HARLAN (Richard). Description of Three Species of the Genus Astacus inhabiting the United States. Medical and Physical Researches, 229 (1835).

HAY (Oliver P.) Description of a New Species of Asellus. Bulletin Illinois State Laboratory of Natural History, i, No. 2, 90-92 (1878).

-Notes on some Fresh Water Crustacea, together with descriptions of two new species. Amer. Naturalist, xvii, 143-146, 241-243 (1882).

HerricK (Clarence L.) A New Cyclops. 5th Report on the Geology of Minnesota, 238, 239 (1877).

-Fresh Water Entomostrica. Amer. Naturalist, xiii, 620$624(1879)$.

Microscopic Entomostraca. Appendix B to the Report on the Geology of Minnesota, 81-123, Plates i-xxi (1879). -Papers on the Crustacea of the Fresh Waters of Minnesot:. 10th Report on the Geolugy of Minnesota, 219254, Plates i-xi (1882).

A New Genus and Species of the Crustacean Family Lyucodaphnidx. Amer. Naturalist, xvi, 1006-1007 (18S2). Heterogenetic Development in Diaptomus. Aner. Naturalist, xvii, 381-389, 499-505 (1883).

A Final Report on the Crustacea of Minnesota, included in the Orders Cladocera and Copepoda. 12th Anuual Report of the Geological Survey of Mimesota (1884). Mud-Inlabiting Crustacea. Bull. Scientific Lab. of Denison University, i, 37-42. Plate ix (1885). 
Metamorphosis and Morphology of Certain Phyllopod Crustacea. Bull. Scientific Lab. of Denison University, i, 16-24. Plates v-viii (1885).

Hubbard (Henry G.) Two Days' Collecting in the Mammoth Cave, with Contributions to a Study of its Fanna. Amer. Entomologist, uew series, i, 34-40, 79-80 (1880). JAMES (Edwin). Note 7 [on the Appearance of Crustacea] to Long's Expedition from Pittsburg to the Rocky Mountains, ii, 336 (1823).

Johnsson (A.) Sveriges Oniscider. Upsala (1858).

JURINE (L.) Histoire des Monocles aux environs de Geneve. With 22 plates. Geneva (1820).

KELLICOTT (David S.) Description of a New Species of $A r$ gulus (A lepidostei). Bull. Buffalo Soc. of Nat. Science, iii, 214-216 (1877), also published in Amer. Jonrnal of Microscopy, iii, 1-3 (1878).

On Certain Crustacea Parasitic on Fishes from the Great Lakes. Proc. Amer. Soc. of Microscopists, i, 53-5\%; 3 plates (18\%9).

Observations on Lerneocera cruciata. Proc. Aner. Soc. of Microscopists, i, 64-68 (1879).

Argulus lepidostei, male. Amer. Jonrnal of Microscopy, iv, 153-155 (1879).

A larval Argulus. North Amer. Entomologist, i, 57 (1880).

-Argulus stizostethii n. s. Amer. Jour. of Microscopy, v, 53-58; plate (1880).

Lerneocera tortua n. s. Proc. Amer. Soc. of Microscopists, ii, 41 (1880).

On Certain Crustaceous Parasites of Fresh Water Fishes. Proc. Amer. Soc. of Microscopists, iv, 75-78 (1882).

KINGSLEY (John S.) Notes on the North American Caridea in the Museum of the Peabody Academy of Science at Salem, Mass. Proc. Phila. Acad. of Science, 1878, 89. List of the North American Crustacea belonging to the sub-order Caridea. Bull. Essex Institute, x, 53-71.

Koch (C. L.) Deutschlands Crustaceen, Arachniden, und Myriapoden. Regensburg (1835-1844). 
KROYER (Henrik). Apus glacialis. Naturhistorisk Tirlsskifift. 2d series, ii. 431-435 (184i).

Bidrag til Kinndskab om inyltekrebsene. Naturhistorisk Tidskifift, ill series, ii, l'lates i-xriii (1563).

Kurz (W.) Dodekas nener ('ladoceren. Wien (15:4).

LE CONTE (John L.) Description of a New Species of Apus. Amals of New York Lycem of Natural History, iv, 1 in $(1545)$.

-Description of New Species of Astacus from Georgia. Proc. Phila. Acad. of Science, vii, 400 (1S5j).

Lereboullet (Anguste). Memoire sur les ('rustaces de la famille des ('loporticles. 10 plates. Strasburg (1S53).

Lesueur (Charles A.) On Three New Species of Parasitic Termes. belonging to the Linnwan Gemns Lernax. Jour. of the Pliila. Acad. of Science, iii, $2 \$ 6$ (1824).

LEYDIG (Franz). Naturgeschichte der Daphniden. 10 plites. Tiibingen (1)60(1).

LiEviN (—). Die Branchiopoden der Danziger Gegend. 11 plates. Danzig (1StS).

LILLGEBORG (Wilhehm). De (rustaceis ex ordinibus tribus Cladocera, Ostracoda et Copepoda in Seania occur. 2i plates. Lumr (1553).

Leptodora hyalina. Öfrersigt af Tetensk.-Akad. Fïrlatudl. 1560. 265, pl. vii.

LINNE (Carl rou). Systema Natura. Tomus i, 12th edition. Stockholm (1766-1\%6\%).

Loven (s.) Om nagra i Vettern och Venern famna Crustacee. Öfrersigt af Vetensk.-Akad. Förhandl., 1S61. 245.

Milne-Edwards (Henri). Histoire Naturelle des ('rustaces. 3 vols. and Atlas (1S38-1840).

MORSE (Edward S.) [Note on the Mode of frowtl of a New Fntomostracons Crustacean]. Proc. Boston Society of Nat. Hist., xi, 404 (1S6S).

MÜLLER (O. Fr.) Entomostraca. sell Insecta Testacea in aquis Dania et Norvegie reperta. 2.) plates. Lipsile (1785).

MÜLLER (P. E.) Dammarks ('ladocera. ('openhagenen (1Stis'). OLSSON (P.) Prodromms Fame ('opepodarmm parasit. Scauldin. Lund (1S6!)). 
PACKARD (Alpheus S., Jr.) On the Crustacea and Insects of the Mammoth Cave. Amer. Naturalist, v, 744-761 (1871).

Preliminary Notice of New North American Phyllopod Crustaceans. Amer. Journal of Science and Arts, 3d series, ii, 108 (1871).

On the Cave Fauna of Indiana. 5th Report of the Peabody Academy of Science, 93-97 (1873).

-Deseriptions of New North American Phyllopoda. 6th Report of the Peabody Academy of Science, 54 (1874). Description of a Lernæan Crustacean obtained by Lient. W. L. Carpenter in 1873, in Colorado. Report of Geological Survey of Territories, 1874 (Hayden), 612. Synopsis of the Fresh Water Phyllopod Crustacea of North America. Report of Geological Survey of Territories, 1874 (Hayden), 613-622, Plates i-iv.

New Phyllopod Crustaceans. Amer. Naturalist, ix, 311 (1875).

Descriptions of new Phyllopod Crustacea from the West. Bulletin of the United States Geological Survey (Hayden ), iii, 171-179 (1877).

- Notes on Phyllopod Crustacea. Amer. Naturalist, xiv, $53(1880)$.

A Monograph of the Phyllopod Crustacea of North America, with remarks on the Order Phyllocarida. 12th Report of the Geological Survey of the Territories (Hayden), ii, 295-593, Plates i-xxxix (1883).

RANDALL (J. W.) Catalogue of Crustacea Brought by Thomas Nuttall and J. K. Townsend from the West Coast of America, etc. Jour. of the Phila. Academy of Science, viii, 106-147, Plates iii-vii (1839).

RYDER (John A.) Description of a New Species of Chirocephalus (Ch. Holmani). Proc. Phila. Acad. of Science, 1879, 148-149.

Description of a new Branchipod. Proc. Phila. Acad. of Science, 1879, 200-202.

SARS (G. O.) Öfversigt af de indenlandske Ferskvands Copepoder (1862).

On en i Sommeren 1862, foretagen zoölogisk Reise i Christianias og Trondhjems stiftor (1863). 
SAY ('Thomas). An Account of the Crustacea of the United States. Journal of the Philadelphia Academy of Science, i, 57-63, 65-80, 97-101, 155-169, 235-253, 313-319, $374-401,423-458$ (1817).

SCHÖDLER (J. E.) De Branchiopoden der Ungegend von Berlin. Berlin (1858).

-Ueber der Lynceiden und Polyphemiden der Umgegend von Berlin (1862).

Nene Beiträge zur Naturgeschichte der Cladoceren (1863).

Cladoceren des Frischen Hoffs (1863).

Zur Naturgeschichte der Daphniden (187\%).

Smith (Sidney I.) Prof. Cope's Cave Crustaceans. Amer. Naturalist, vii, 245 (1873).

The Crustacea of the Fresh Waters of the Northern United States. Report of the U. S. Commissioner on Fish and Fisheries for 1872-73, 637-665, Plates i-iii.

Sketch of the Invertebrate Fauna of Lake Superior. Report of the U. S. Commissioner of Fish and Fisheries for $1872-73,690-70 \%$.

Report on the Amphipod Crustaceans [collected by Lient. W. I. Carpenter in 1873, in Colorado]. Seventh Report of U. S. Geological Survey of the Territories (Hayden), 608-611, 2 plates (1874).

The Crustaceans of the Caves of Kentucky and Indiana. Amer. Journal of Science and Arts, 3d series, ix, 4\%6, $477(1875)$.

SMITH (S. I.) and VERRILL (A. E.) Notice of Invertebrata dredged in Lake Superior in 1871. Amer. Journal of Science and Arts, 3d series, ii, 448 (18\%1).

Strmpson (William). Notes on North American Crustacea, No. I. Annals of New York Lyceum of Natural History, vii, 40-93, Plate i. No. II, l. c. vii, 176-246, Plate ii. No. III, 1. c. x. 92-136.

On the Crustacea and Echinodermata of the Pacific Shores of North America. Boston Journal of Natural History, vi, 444-532, Plates xviii-xxiii (1857). Also Abstract in Proceeding Boston Society of Natural IIistory, vi, 84 (1857). 
On the Deep Water Famma of Lake Michigan. Amer. Naturalist, iv, 403 (1870).

Strauss (H. E.) Memoire sur les Daphnia de la classe des Crustaces. Mem. du Mus. d'Hist. Nat., v and vi (1819$1820)$.

Strents (Thomas Hale). Cambarus conesi. Bulletin U. S. Geological Survey of the Territories (Hayden), iii, 803 (1877).

STUXBERG (Anton). Om Ñord-Amerikas Oniscider. Öfversigt af Vetensk.-Akad. Förhandl., No. 2, 43-63 (1875).

VERRILL (Addison E.) Descriptions of Some New American Phyllopod Crustacea. Amer. Journal of Science and Arts, 2il series, xlviii, 244-254 (1869).

-New Localities of Artemia. Amer. Journal of Science and Arts, $2 d$ series, xlviii, 480 (1869).

Observations on Phyllopod Crustacea of the Family Branchipida with Descriptions of Some New Genera and Species from America. Proceedings of the Amer. Association for the Advancement of Science for 1869.

VERRILL (A. E.), SMITH (S. I.), and HARGER (O.) Report upon the Invertebrate Animals of Vineyard Sound and the Adjacent Waters. Report of the U.S. Commissioner of Fish and Fisheries for 1871, 295-778, Plates ixxxviii [i-ix on Crustacea].

Wright (R. Ramsay). Notes on American Parasitic Copepoda, No. 1. Proceedings Canadian Institute, n. s. i, No. 3. 243-254.

On a Parasitic Copepod of the Clam. Amer. Naturalist, xix, 118-124 (1885).

ZADDACH (E. G.) Holopedium gibberum, ein nenes Crust. aus der Familie der Branchiopoden. Archiv für Naturgeschichte, 1855, plates viii, ix. 


\section{INDEX OF GENERA.}

(Families in Swall CAPITAL, orders in CAPITALS.)

\begin{tabular}{|c|c|}
\hline chtheres. & \\
\hline ctoniscus ................... & Crangonyx ...... \\
\hline croperus.................... & Crepidocercus .... \\
\hline llorchestes ............... & Cyclops .............. \\
\hline lloniseus............... & CyclOPIDA.......... \\
\hline lona .................... & Crpridid . ......... \\
\hline Ionella ..................... & Cypris .............. \\
\hline lonopsis ...................... & Bythernia ......... \\
\hline HIPODA..... & Daphnella.. ....... \\
\hline PODID.T.......................... 3 & Daplınia.......... \\
\hline pus ............................. & DAPHNIDE...... \\
\hline gulus .............. & САРОDА.... \\
\hline ULID.E............... & omus........... \\
\hline rmadillidium, ................... & hura .......... \\
\hline adillo ........................... $3 t$ & ASILID_E........ \\
\hline aia.......................... & (............... \\
\hline 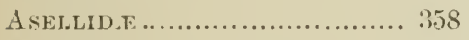 & ladia .......... \\
\hline ..................... & cia ............ \\
\hline 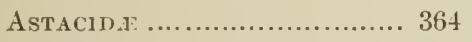 & ereus ................. \\
\hline s......................... & ARID.E. ........ \\
\hline aa $\ldots . . . . . . . . . . . . . . . .$. & is .................... \\
\hline$\pi$ & eberis ............... \\
\hline tal.... & CTIDE........ \\
\hline DID $\mathrm{X}$ & SDID $\mathrm{x} . . . . . .$. \\
\hline hipus ...................... & lium.......... \\
\hline lotea .......................... & is ................ \\
\hline Calanidæe........................... & ODA $\ldots \ldots \ldots \ldots \ldots$ \\
\hline E . ........................ & ura .................. \\
\hline $\mathrm{s}$.................... & .................. \\
\hline Camptocercus..................... & ocera ............... \\
\hline nom & CERIDE........... \\
\hline tocamntus ............. & reopoda ........... \\
\hline renus & LERN EOPODID E......... \\
\hline $11 a . . .$. & heirus ....... \\
\hline ephalus ..... & iurus.............. \\
\hline & Leptorlora ............. \\
\hline CLA DOCERA ..... & LEPTODORID A: ......... \\
\hline
\end{tabular}




\begin{tabular}{|c|c|}
\hline Leydigia.......................... 345 & Palæmonetes ..................... 374 \\
\hline Ligia ............................. 360 & PALEMONID \\
\hline Ligidium ........................ 361 & Pen \\
\hline Limnadia......................... 352 & Penæus....................... \\
\hline Limnadella...................... 352 & Philoscia ....................... \\
\hline LimNadide ..................... 350 & PHYLLOPODA........... \\
\hline Limnetis .......................... 350 & Pleuroxus ........... \\
\hline Limnocalanus ..................... 329 & Polyphemide ...... \\
\hline 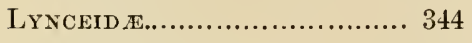 & Polyphemus .............. \\
\hline Lyncodaphnia.................... 344 & Pontoporeia ................ \\
\hline LYNCODAPHNID 2 ................ 343 & Porcellio ............. \\
\hline LYSIANASSID $\approx \ldots \ldots \ldots \ldots \ldots \ldots \ldots \ldots \ldots$ & Pseudo-sida ........... \\
\hline Macrothrix...................... 343 & Rhinoryctes ........... \\
\hline Mancasellus ...................... 359 & Scapholel \\
\hline 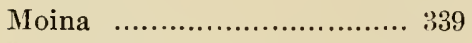 & 363 \\
\hline Monospilus....................... 349 & Sida .................. \\
\hline MYSID E .......................... 364 & SIDIDE.............. \\
\hline Mysis ............................ & Simocephalus .............. \\
\hline Notadromus ...................... 337 & Sphærillo ........................ \\
\hline ONISCID & Streptocephalus .. .............. \\
\hline Oniscus........................... 361 & Styloniscus ..... ................ 364 \\
\hline ORCHESTID E...................... 358 & Tachidius...................... 333 \\
\hline Osphranticum ................... 32 & Thamnocephalus............... 355 \\
\hline OSTRACODA .................. 336 & Trichoniscus .................. 364 \\
\hline & \\
\hline
\end{tabular}




\section{ERRATA.*}

Page 5 , line 3 of table, second column, for 39 read 38 ; line 6 , second column for 121 read 120.

Page 9, line 17, for conjunction read conjugation.

Page 21, line 13, for Kicciacir read Ricciacea.

Page 67 , line 17 from bottom, for fraligifolia read fragilifolia.

Page 123, line 4 from bottom, and page 126, line 1, for T'richolece read Trichocolea.

Page 126, line 2, for Tricholea read Trichocolea.

Page 17\%, line 16, for Lecythia read Lecythea.

Page 333, line 1, after Tachidius add Lilljeb.

Page 338, under Daphnella brachyura, line 16, insert Hab.-Massachusetts (Birge), Minnesota (Herrick).

Page 340, line 5, for Scapuoleberis read Scapholeberis.

Page 389, line 7 from bottom, for carpogonium read sporocarp ; lines $9,12,15$, for ö̈gonium read carpogonum.

Page 391, line 1, for Cessatii read Cesatii.

Page 400, line 4, for Myceliumin conspicnous read Mycelium inconspicuous ; line 14, for coleosporium read Coleosporium.

Page 401, line 9, for connatus read connata; line 12, for Taraxicnm read Taraxacum.

Page 408, line 15, for macrocarpa read macrospora; line 18, for $\mathrm{Hy}$ pohyllous read Hypophyllous.

Pages 470 and 471, head of column 11, for cyprinella read cyprinellus.

Page 503, lines 8, 14, and 17, for cyprinella read cyprinellus.

* For additional errata see page 247. 